J. Nat. Hist. Mus. Vol. 30, 2016-18

$55-84$

\title{
A checklist of flowering plants of Panchase Protected Forest, Kaski district, central Nepal
}

\author{
Prabin Bhandari $^{1} \bowtie$, Shanta Budhamagar ${ }^{1}$ and Krishna K. Shrestha ${ }^{1}$ \\ ${ }^{1}$ Central Department of Botany, Tribhuvan University, Kirtipur, Kathmandu, Nepal \\ prabinkb02@gmail.com
}

\begin{abstract}
Panchase Protected Forest (PPF), situated at the junction of three districts Kaski, Syangja and Parbat with an elevation range of $900-2517 \mathrm{~m}$ representing mid hills of Nepal. This study was carried out with an aim of documenting the flowering plant diversity of the Panchase Protected Forest, Kaski district, comprising three VDCs, Bhadaure Tamage, Chapakot and Pumdi Bhumdi. General survey, covering all seasons was organized (from June 2014 to May 2015) for collecting the plant species. Published literatures and herbarium specimens were also consulted for documenting the species. Identification of the collected specimens was made through consulting relevant literatures, herbarium specimens and expert consultation. A total of 613 species belonging to 393 genera and 111 families have been documented from this area. The dominant family was Orchidaceae (49 genera and 125 species) followed by Poaceae (25 spp.), Asteraceae (25 spp.) and others. Six endemic plants, Begonia flagellaris (Begoniaceae), Eria pokharensis, Gastrochilus nepalensis, Oberonia nepalensis, Odontochilus nandae and Panisea panchaseensis (Orchidaceae) were also reported. Similarly, present research revealed two taxa as new record for flora of Nepal, Dischidia bengalensis Colebr. (Apocynaceae) and Phreatia elegans Lindl. (Orchidaceae). The present findings suggest that PPF is one of the hotspot for floral diversity representing mid hills of Nepal and this pristine habitat for orchid should be developed as 'Orchid Sanctuary'.
\end{abstract}

Keywords: endemic, flora, mid hills, new record, orchidaceae

\section{INTRODUCTION}

Mid hills of Nepal are the depositories of large number of plant species as maximum species richness lies in this range (1500-2500m) (Vetaas \& Grytness, 2002). Panchase is an important midmountain ecological zone of Nepal (Bhattarai et al., 2012) and is considered as a biological corridor between Chitwan National Park and Annapurna Conservation Area. Panchase mountain harbors diverse ecosystem: forest, shrubland, wetland, rangeland and agriculture; Schima-Castanopsis and temperate broad leaved forest predominant the region (GoN, 2013; Maren et al., 2013). This forest housed rare, threatened and endemic plant species including six endemic and three threatened orchid species (Bajracharya et al., 2003; Subedi et al., 2007, 2011; Maren et al., 2013; Raskoti, 2015; Raskoti \& Kurzweil, 2015). Furthermore, Panchase is home to number of endangered wildlife species including the Himalayan black bear (Ursus thibetanus), the common leopard (Panthera pardus), and eight species of bats (Aryal \& Dhungel, 2009; Malla et al., 2013). Considering the biological significance of this 
region, Government of Nepal, MoFSC, has declared the forest as "Panchase Protected Forest" in 2012 (DoF, 2012).

Botanical exploration in Panchase starts since early 1950s. Stainton and his colleagues collected several plant specimens along Pokhara to Baglung in 1954 (Stainton, 1972). During that visit, some collections were from Mt. Panchase as registered in Flora of Nepal database (www.floraofnepal.org). Joshi and Amatya collect 1611 plant specimens from Central Nepal along the route Butwal-Pokhara in 1973 (Rajbhandari, 2002); a few plant specimens collected from Panchase during the expedition of Joshi and Amatya have been listed in Flora of Nepal database. Similarly, hundreds of plant specimens were collected and deposited in Herbarium of the University of Tokyo, Japan (TI) in 1999 by a Herbal expedition team of Kanazawa University, Japan, from Panchase region (see TI Herbarium database).

Koirala (1998) reported 306 plant species including some medicinal plants from Panchase. Subedi (2002) reported 170 species of orchids around Pokhara valley of which 98 species are represented from Panchase. Bajracharya et al. (2003) reported an endemic orchid 'Eria pokharensis Bajrach., Subedi \& K.K. Shrestha' from Lumle. Subedi et al. (2007) reported 112 species of Orchids from Panchase forest. Subedi et al. (2011) recorded an endemic orchid 'Panisea panchaseensis Subedi' from Panchase. Raskoti and Kurzweil (2015) recorded an endemic orchid species 'Odontochilus nandae Raskoti \& H. Kurzweil' from Panchase. Similarly, Raskoti (2015) reported another endemic orchid 'Gastrochilus nepalensis Raskoti' from Panchase mountain. Thus this paper attempts to compile the previous works along with the present study, listing the flowering plants of Panchase Protected Forest, Kaski district, Central Nepal.

\section{MATERIALS AND METHODS}

\section{Study area}

The Panchase area is situated in the mid hills of central Nepal, at the junction of three districts, Kaski, Parbat and Syangja. It lies at latitudes between $28^{\circ} 12^{\prime}$ and $28^{\circ} 18^{\prime} \mathrm{N}$ and longitudes $83^{\circ} 45^{\prime}$ and $83^{\circ} 57^{\prime} \mathrm{E}$. The total area of the Panchase forest covers $45.93 \mathrm{Km}^{2}$ of which Kaski district shares $23 \mathrm{Km}^{2}$, comprising three VDCs, Bhadaure Tamage, Chapakot and Pumdi Bhumdi. Altitude ranges from $900 \mathrm{~m}$ to $2517 \mathrm{~m}$. Panchase region possesses extreme steep slope to flat area with different terraces of terrain, and exists on a geological rock of mid land group. Climate is subtropical at lower altitudes and moist temperate above $2000 \mathrm{~m}$. Many rivers and tributaries originated from Panchase, among them Phewa lake is the main reservoir.

Wide climatic variation, steep vertical gradient allows a high diversity of ecosystem (wetland, forest and grassland) in Panchase region. Panchase area consists of hill Sal forest at base succeeding Schima-Castanopsis forest, Alder forest, lower temperate oak forest, Oak Laurel forest and Rhododendron-Taxus forest in the top. 

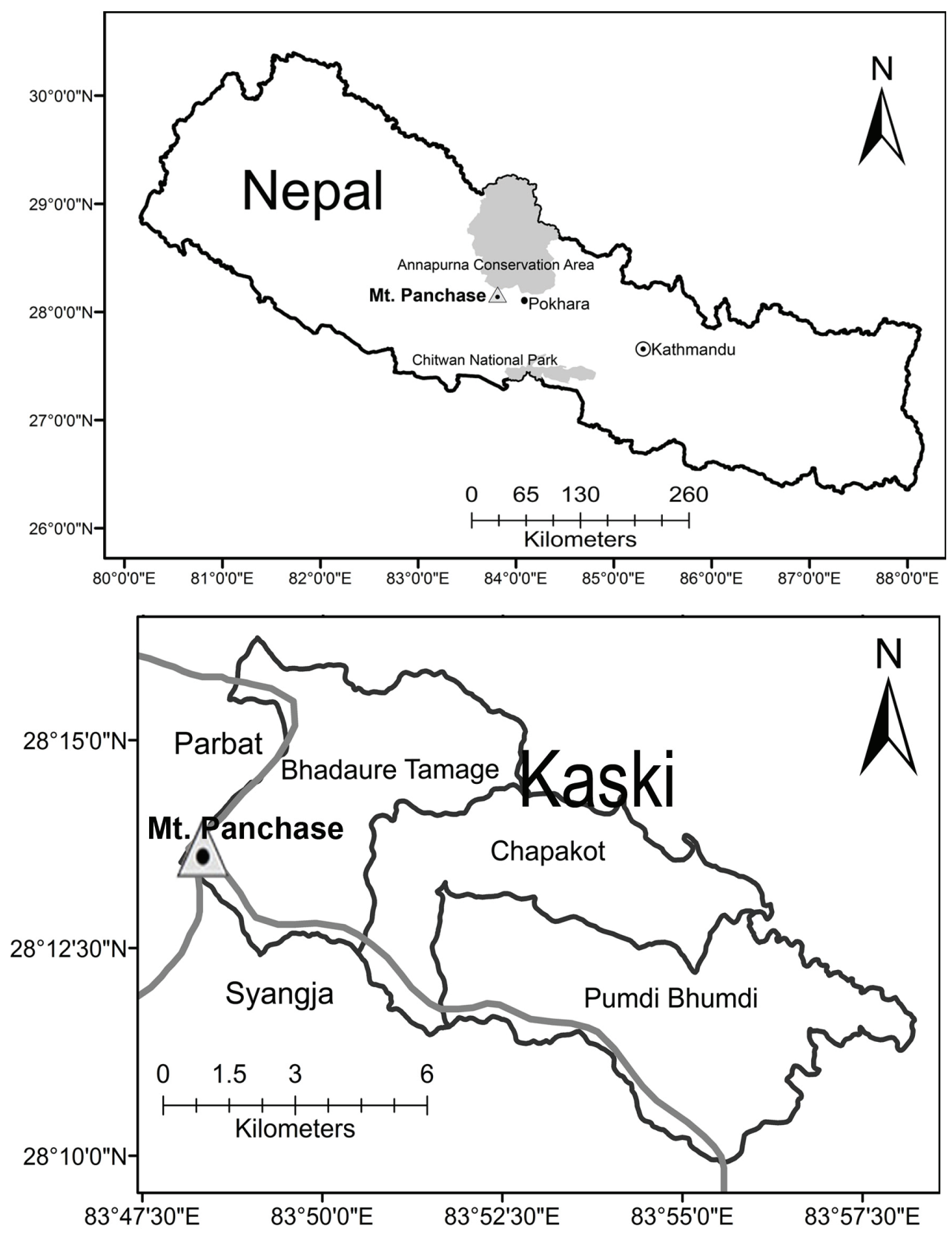

FIG. 1. Location of Panchase in Nepal, enlarged portion showing the studied VDCs. 


\section{Data collection}

Field visits were carried out from June 2014 to August 2015 within the elevation range of 900$2500 \mathrm{~m}$ covering all the habitat types and vegetation zones, wherever possible. Previously collected specimens from Panchase, deposited at Tribhuvan University Central Herbarium (TUCH) and National Herbarium and Plant Laboratories (KATH) were also studied. Information on herbarium specimens housed outside Nepal was obtained through online information on herbaria [Herbarium of the University of Tokyo, Japan (TI) database, www.plants.jstor.org, www.floraofnepal.org. The present checklist also includes the collection of A. Subedi from 1998 to 2007, based on Subedi et al. (2007). The collected plant specimens were identified using relevant taxonomic literatures: Hooker (1872-1897), Flora of Bhutan (Grierson \& Long, 1983-2001; Noltie, 1994-2000; Pearce \& Cribb, 2002), Malla et al. (1986), Wu et al. (19942008), Press et al. (2000), Raskoti (2009), Watson (2011). Doubtful specimens were identified through expert consultation. Final confirmation of identified species was done by comparing the specimens deposited at $\mathrm{KATH}$ and $\mathrm{TUCH}$ and with the images of specimens deposited at Herbarium of University of Tokyo, Japan ( $\mathrm{TI}$ ), Royal Botanic Garden, Kew, England (K) and Royal Botanic Garden, Edinburgh, England (E). The identified specimens were deposited at TUCH and KATH.

For the nomenclature and author citation of plant species, TROPICOS (www.tropicos.org) and 'The Plant List' (www.theplantlist.org) were followed. The families are based on APG III (2009). For reader's convience, the checklist (table1) is arranged alphabetically.

\section{RESULTS AND DISCUSSION}

The flora of Panchase Protected Forest Protected Forest was composed of a total of 613 species under 393 genera and 111 families of flowering plants (table 1), which includes the present collection, published literature and past collection housed in national (KATH and $\mathrm{TUCH}$ ) and international herbaria (BM, K, TI). Three families represent gymnosperms with 4 genera and 5 species. Angiosperms were represented by 108 families with 608 species belonging to 389 genera. Among 613 flowering plants, $64 \%$ (395 spp.) of total species was represented by herbs, $15 \%$ (92 spp.) by trees, shrubs by $12 \%$ (73 spp.) and $9 \%$ (53 spp.) by climbers.

Orchidaceae was the dominant family with 49 genera and 125 species, followed by Poaceae, Asteraceae, Lamiaceae, Fabaceae, Rubiaceae, Cyperaceae, Urticaceae, Acanthaceae, Araceae, Apocynaceae, Moraceae and others. The largest genus recorded was Bulbophyllum comprising 18 species, followed by Dendrobium (13 spp.), Ficus (10 spp.), Impatiens with 8 spp., etc. The other larger genera were Arisaema, Coelogyne, Cymbidium, Oberonia, Polygonum, etc. 


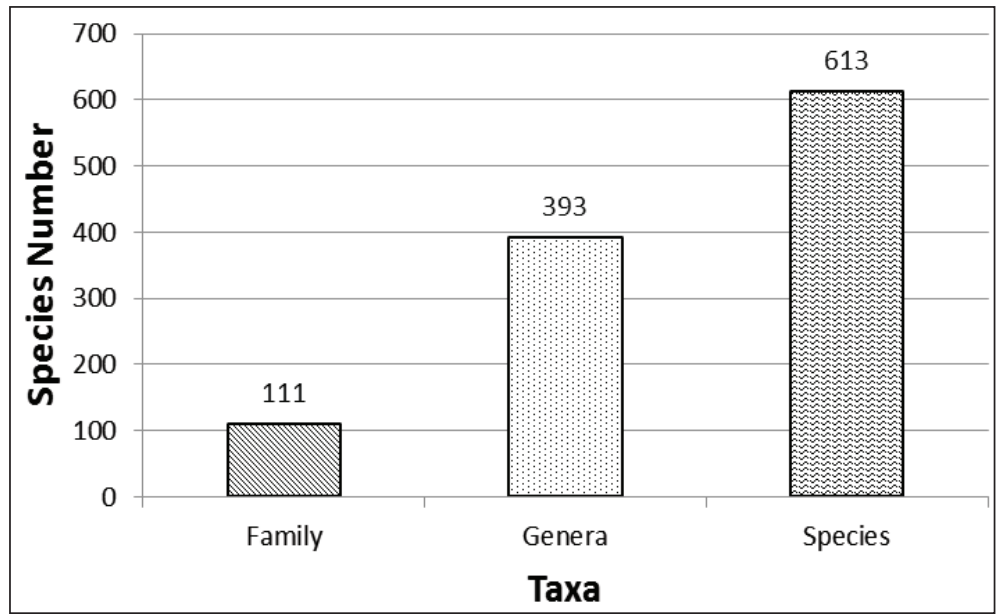

FIG. 2. Total number of taxa in Panchase protected forest.

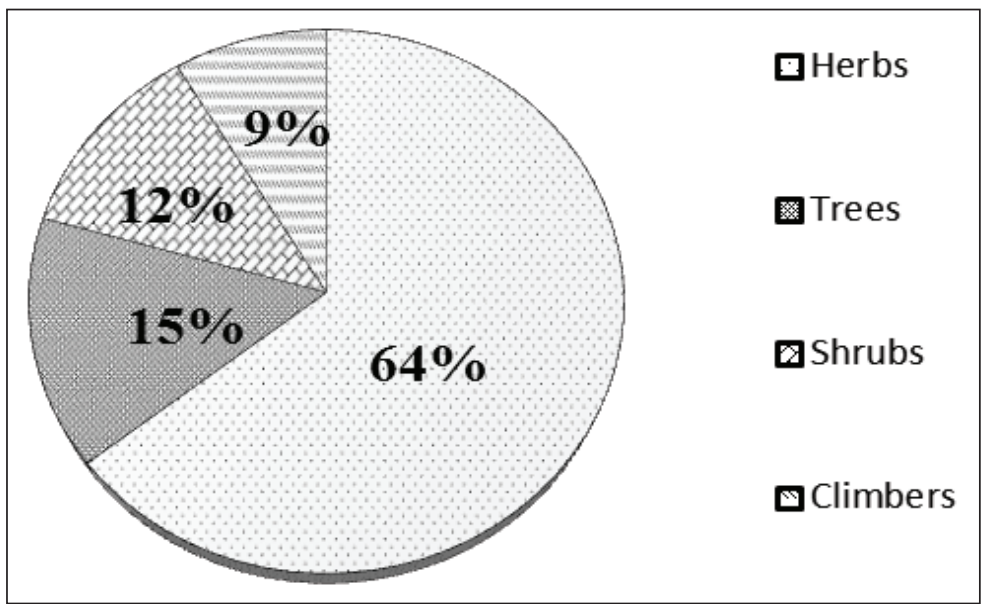

FIG. 3. Percentage of life forms of Plant species from Panchase region.

Panchase is reported to have 6 endemic plant species of Nepal, of which the four species: Eria pokharensis, Gastrochilus nepalensis, Odontochilus nandae, Panisea panchaseensis and (Orchidaceae) are local endemic (Bajracharya et al., 2003; Subedi et al., 2011; Raskoti, 2015; Raskoti \& Kurzweil, 2015) and not found beyond Panchase and the rest two [Begonia flagellaris H.Hara (Begioniaceae), Oberonia nepalensis (Orchidaceae)] are region endemic and distributed in Central Nepal (Shakya \& Chaudhary, 1999; Rajbhandari \& Adhikari, 2009; Rajbhandari \& Dhungana, 2010), in and around Panchase region.

So far, the previous works are concerned, Koirala (1998) has given a list of 310 plant species from Panchase, of which no voucher specimen could be traced. Some species reported by Subedi et al. (2007) ( ${ }^{*}$ sign in checklist) are of doubtful occurrence as no voucher specimens could be traced and 
they have not been collected during the present work. Similarly, we could not discover the two species (Tsuga dumosa (D.Don) Eichler and Pedicularis gracilis Wall. ex Benth.) which Stainton collected about 60 years ago from Panchase region, moreover flora of Nepal database has registered these species collected from Panchase as housed in BM and E (www.floraofnepal.org).

Panchase is the natural habitat of nearly one third orchids of Nepal, and this pristine habitat for orchid should be developed as "Orchid Sanctuary". The overall plant diversity of PPF shows the area's ability supporting rare, endemic, threatened as well as common species. Thus PPF can be regarded as one of the hotspot of plant diversity in central Nepal.

\section{New addition to flora of Nepal}

The present study revealed two genera including the respective species as new addition to the flora of Nepal, namely Dischidia bengalensis Colebr. [Apocynaceae] (Bhandari \& Shrestha, 2016) and Phreatia elegans Lindl. [Orchidaceae] (Bhandari et al., 2015).

\section{ACKNOWLEDGMENTS}

Authors are thankful to National Herbarium and Plant Laboratories (KATH) and Tribhuvan University Central Herbarium ( $\mathrm{TUCH}$ ) for allowing us to examine herbarium specimens. We are also grateful to local peoples of Panchase region for their kind cooperation during the course of study.

\section{REFERENCES}

APG III (2009) An Update of the Angiosperm Phylogeny Group classification for the orders and families of flowering plants: APG III. Bot J Linn Soc 161: 105-121.

ARYAL, A; DHUNGEL, S K (2009) Species diversity and distribution of bat in Panchase region of Nepal. Conservation and Information Network of South Asia 1(1): 11-14.

BAJRACHARYA, D M; SUBEDI, A; SHRESTHA, K K (2003) Eria pokharensis sp. Nov. (Orchidaceae); A new species from Nepal Himalaya. J Orchid Soc India 17(1-2): 1-4.

BHANDARI, P; SHRESTHA, K K (2016) Dischidia bengalensis Colebr. (Apocynaceae)- a new record for the flora of Nepal. The Japanese Journal of Botany 91 (5): 310-313.

BHANDARI, P; RASKOTI, B B; SHRESTHA, K K (2015) Pheretia elegans Lindley (Orchidaceae)- a new record for flora of Nepal. Pleione 9(2): 365-368.

BHATTARAI, K R; MAREN, I E; CHAUDHARY, R P (2012) Medicinal plant knowledge of the Panchase Regon in the middle hills of the Nepalese Himalayas, Banko Janakari 21(2): 31-39.

DoF (2012) Panchase protected forest management plan. Department of Forest (DoF), Ministry of Forest and Soil Conservation (MoFSC), Government of Nepal, Kathamndu, Nepal.

GRIERSON, A J C; LONG, D G (1993-2000) Flora of Bhutan, Vol. 1, Part 1-3; Vol. 2, Part 1-3. Royal Botanic Garden, Edinburgh and Royal Government of Bhutan.

GoN (2013) Ecosystem and ecosystem services of Panchase: an overview. Government of Nepal, Ministry of Forest and Soil Conservation, Kathmandu, Nepal.

HOOKER, J D (1872-1887) Flora of British India. Vol. I-VII. Reev and Company, London, UK.

KOIRALA, R A (1998) Botanical diversity within the project area of Machhapuchhre Development Organization, Bhadaure/Tamage VDC, Kaski district. A baseline Survey for Machhapuchhre Development 
Organization (MDO), Bhadaure/Tamage, Kaski district, Nepal.

MALLA, D B; ARYAL, A; KUWOR, K J (2013) Distribution and food resources of Asiatic black bear (Ursus thibetanus) and human-bear conflict in the Panchase protected forest of Nepal. International Bear News 22(2): 33-35.

MALLA, S B; RAJBHANDARI, S B; SHRESTHA, T B; ADHIKARI, P M; ADHIKARI, S R; SHAKYA, P R; (1986) Flora of Kathmandu Valley. Bull. Dept. med. Plants Nepal No.11. His Majesty's Government, Thapathali, Kathmandu, Nepal.

MAREN, I E; BHATTARAI, K R; CHAUDHARY, R P (2013) Forest ecosystem services and biodiversity in contrasting Himalayas forest management services. Environmental Conservation 41(1): 73-83.

NOLTIE, H J (1994-2000) Flora of Bhutan, Vol. 3, Part 1-2, Royal Botanic Garden, Edinburgh and Royal Government of Bhutan.

PEARCE, N R; CRIB, P J (2002) Flora of Bhutan. Vol. 3, Part 3. Royal Botanic Garden, Edinburgh.

PRESS, J R; SHRESTHA, K K; SUTTON, D A (2000) Annotated checklist of the flowering plants of Nepal. The Natural History Museum, London, UK.

RAJBHANDARI, K R (2002) Flora of Nepal: 200 years's march. In NOSHIRO, S; RAJBHANDARI, K $\mathrm{R}$ (eds) Himalayn botany in the twentieth and twenty-first centuries. The Society of Himalayan Botany, Tokyo, Japan.

RAJBHANDARI, K R ; ADHIKARI, M K (2009) Endemic flowering plants of Nepal, Part 1. DPR Bull. Sp. Publication No.1, DPR, Thapathali, Kathmandu, Nepal.

RAJBHANDARI, K R; DHUNGANA, S K (2010) Endemic flowering plants of Nepal, Part 2. DPR Bull. Sp. Publication No.2, DPR, Thapathali, Kathmandu, Nepal.

RASKOTI, B B; KURZWEIL, H (2015) Odontochilus nandae (Orchidaceae; Cranichidae; Goodyerinae), a new species from Nepal. Phytotaxa 233(3): 293-297.

RASKOTI, B B (2009) The orchids of Nepal. Quality Printers, Kathmandu, Nepal.

RASTOKI, B B (2015) A new species of Gastrochilus and new records for the orchids of Nepal. Phytotaxa 233(3): 179-184.

SHAKYA, L R; CHAUDHARY, R P (1999) Taxonomy of Oberonia rufilabris (Orchidaceae) and allied new species from the Himalaya. Harvard Papers in Botany 4(1): 357-363.

STAINTON, J D A (1972) Forest of Nepal. John Murray, London, UK.

SUBEDI, A (2002) Orchids around Pokhara valley of Nepal. LI-BIRD Occasional Paper No.: 1. Local Initiatives for Biodiversity, Research and Development (LI-BIRD), Pokhara, Nepal.

SUBEDI, A; CHAUDHARY, R P ; VERMEULEN, J J ; GRAVENDEEL, B (2011) Panisea panchaseensis sp. Nov. (Orchidaceae) from central Nepal. Nord $J$ of Bot 29: 361-365.

SUBEDI, A; SUBEDI, N; CHAUDHARY, R P (2007) Panchase Forest: an extraordinary place for wild orchids in Nepal. Pleione 1: 23-31.

VETAAS, O R; GRYTNES, J A (2002) Distribution of vascular plant species richness and endemic richness along the Himalayan elevation gradient in Nepal. Global Ecol Biogeogr 11: 291-301.

WATSON, M F; MKIYAMA, S; IKEDA, H; PENDRY, C; RAJBHANDARI, K R; SHRESTHA, K K (eds) (2011) Flora of Nepal, Vol. 3. The Royal Botanic Garden, Edinburgh, UK.

WU, Z; RAVEN, P H; HONG, D (1994-2008) Flora of China, Vol 4, 8, 11, 13, 14, 16, 18, 19, 20, 22, 24, 25. 


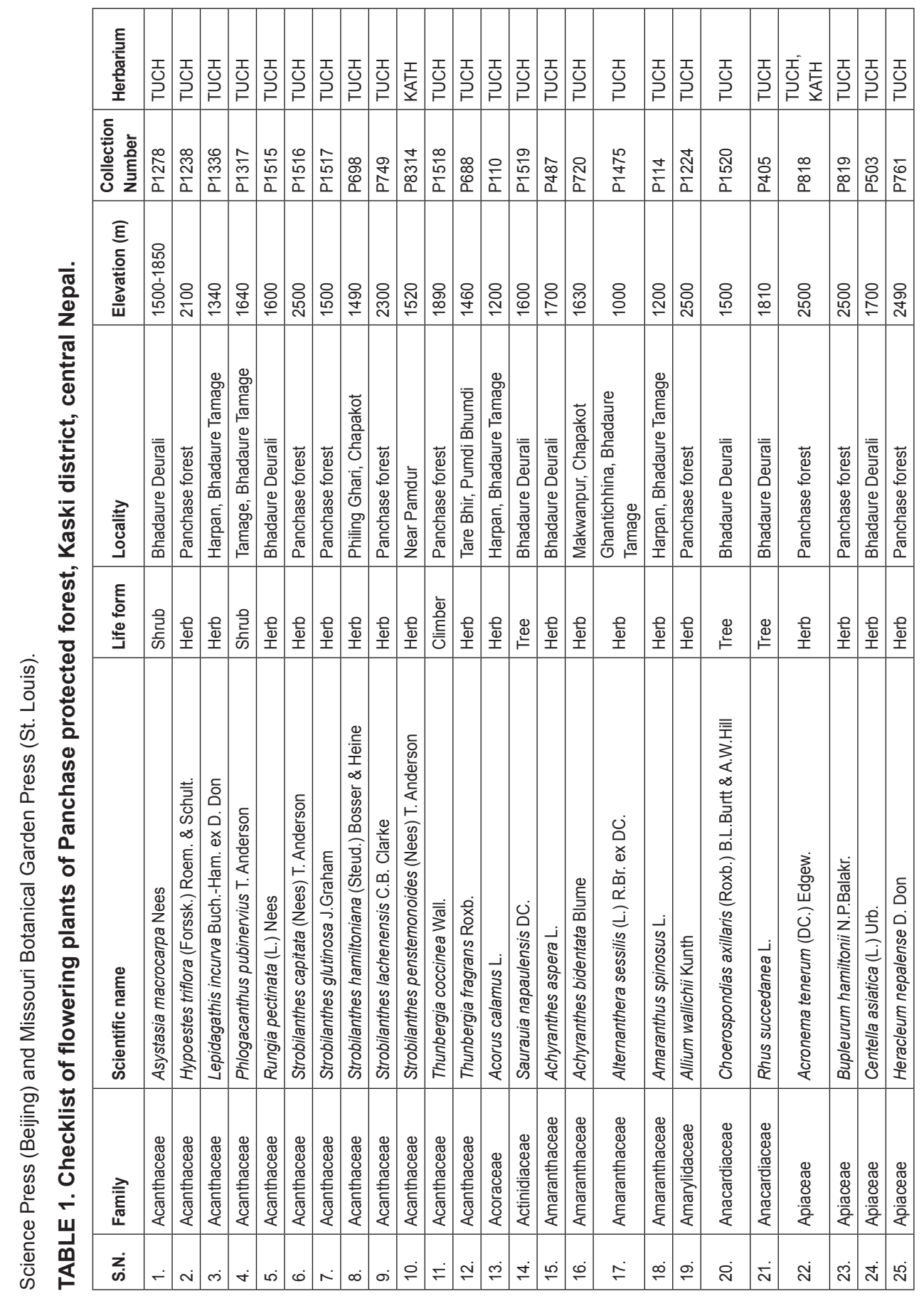




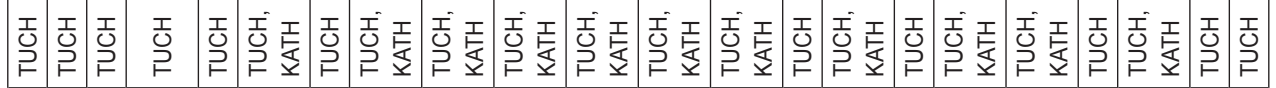

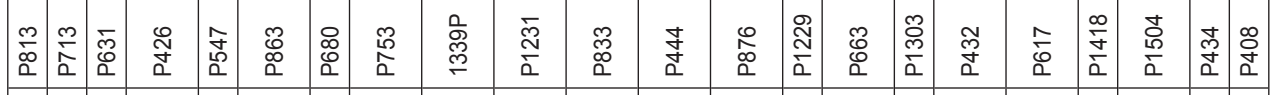

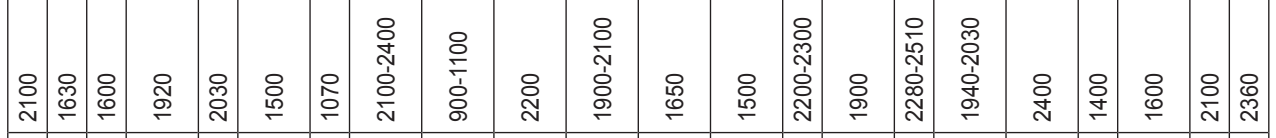

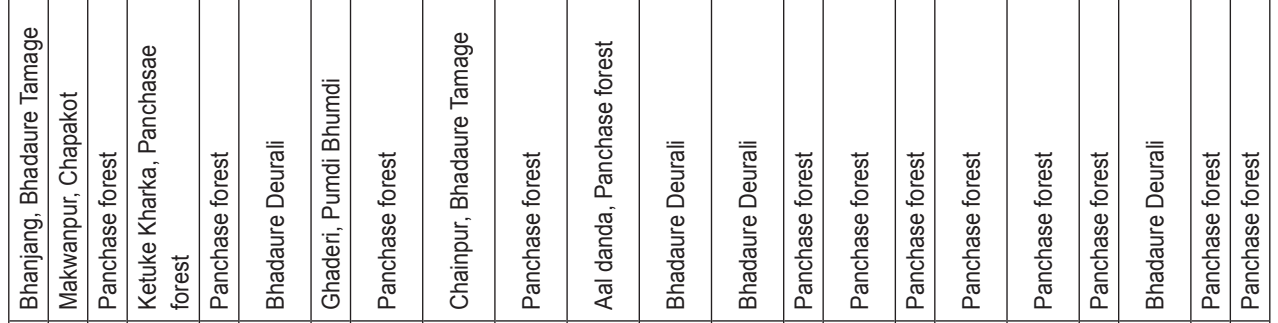

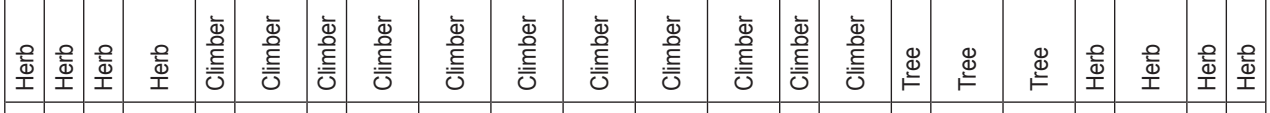

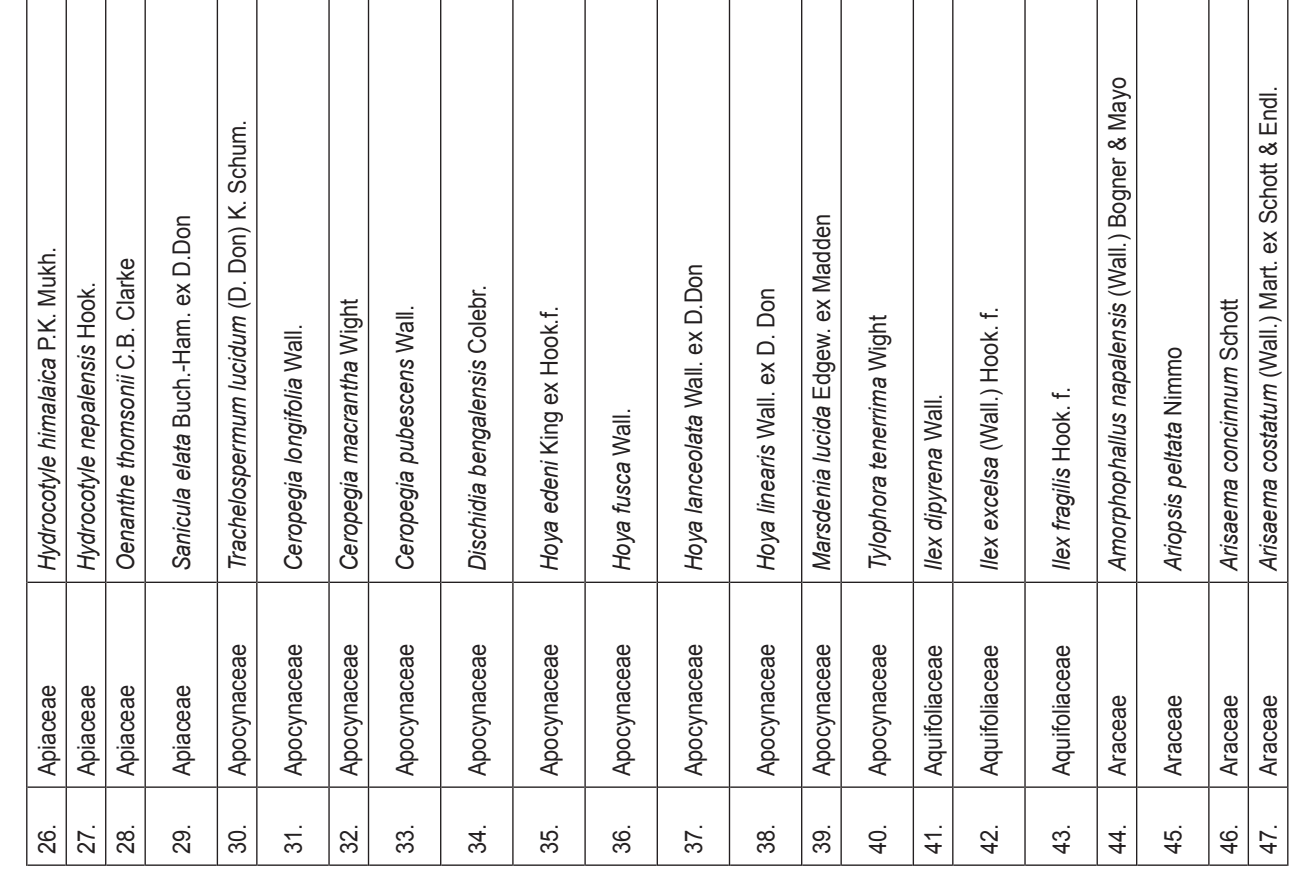




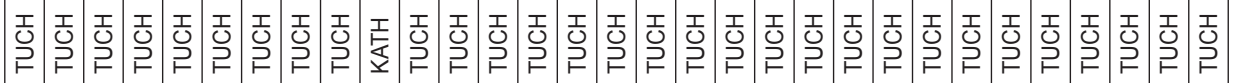

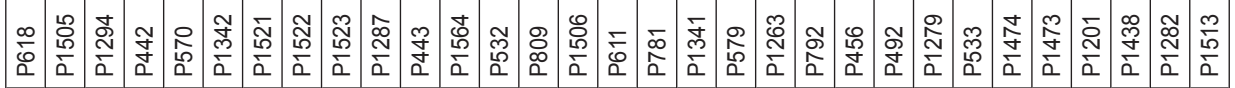

ํํำ

怘

은

은 온

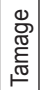

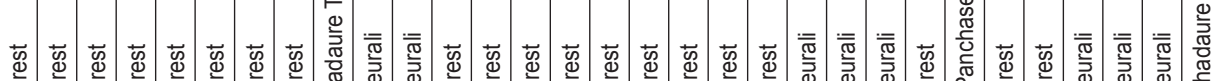

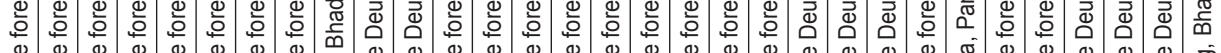

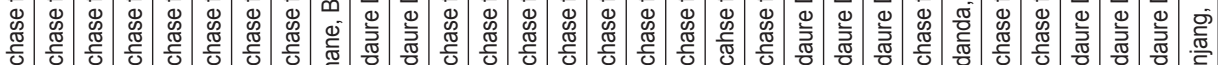

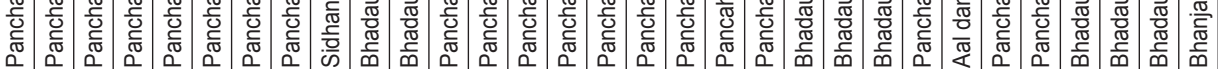

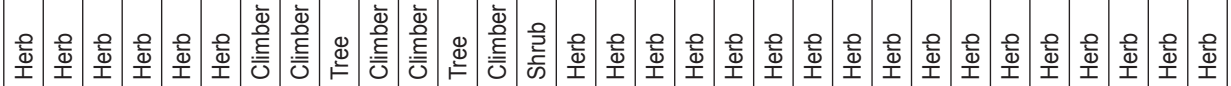

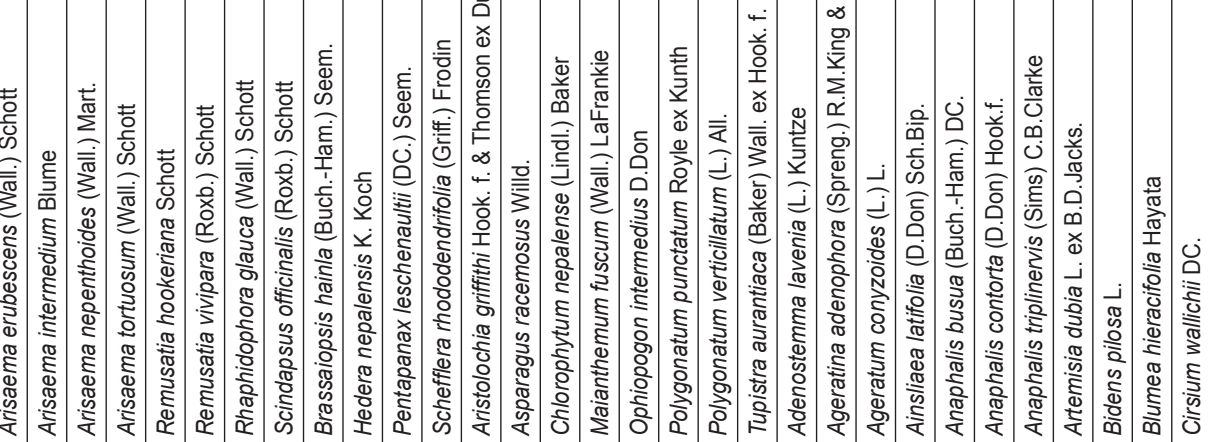

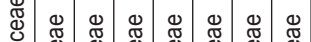

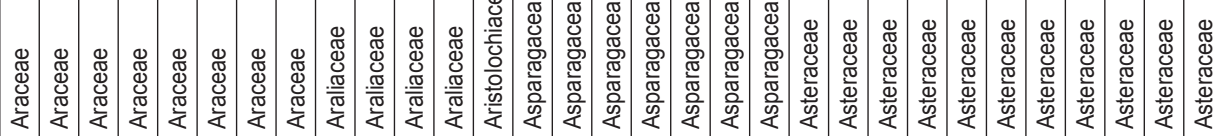

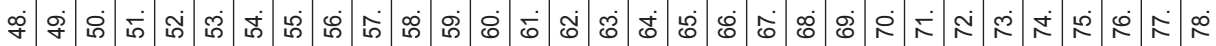




\begin{tabular}{|c|c|c|c|c|c|c|c|c|c|c|c|c|c|c|c|c|c|c|c|c|c|c|c|c|c|}
\hline $\begin{array}{l}\mathrm{I} \\
\mathrm{O}\end{array}$ & $F$ & $\begin{array}{l}\mathrm{I} \\
\mathrm{U} \\
\mathrm{P} \\
\end{array}$ & $\begin{array}{l}\mathrm{I} \\
\mathrm{O} \\
\mathrm{P}\end{array}$ & I & $\begin{array}{l}\mathrm{I} \\
\mathrm{U} \\
\mathrm{P}\end{array}$ & $\begin{array}{l}\mathrm{T} \\
\mathrm{O} \\
\mathrm{P}\end{array}$ & $\begin{array}{l}\mathrm{T} \\
\mathrm{U} \\
\mathrm{P} \\
\end{array}$ & 겅 & 杢 & 杢 & I & I & $\begin{array}{l}\mathrm{I} \\
\mathrm{U} \\
\mathrm{P}\end{array}$ & $\mid \begin{array}{l}\mathrm{T} \\
\mathrm{U} \\
\mathrm{P}\end{array}$ & $\begin{array}{l}\mathrm{I} \\
\mathrm{U} \\
\mathrm{P} \\
\end{array}$ & $\begin{array}{l}\mathrm{I} \\
\mathrm{J} \\
\mathrm{P} \\
\end{array}$ & 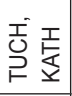 & $\begin{array}{l}\mathrm{I} \\
\mathrm{U} \\
\mathrm{P}\end{array}$ & 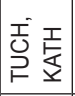 & 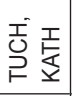 & 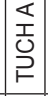 & 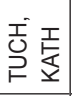 & \begin{tabular}{|l}
$I$ \\
U \\
\\
\end{tabular} & 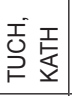 & $\begin{array}{l}\text { I } \\
\text { ㄱ } \\
\text { P }\end{array}$ \\
\hline$\stackrel{\infty}{\frac{m}{\alpha}}$ & \begin{tabular}{|l}
$\bar{\infty}$ \\
$\stackrel{\infty}{\alpha}$ \\
$\bar{\alpha}$
\end{tabular} & $\begin{array}{c}\tilde{N} \\
\hat{\alpha} \\
\alpha\end{array}$ & $\begin{array}{l}\bar{\Xi} \\
\varnothing \\
\alpha\end{array}$ & $\frac{\bar{T}}{\bar{\alpha}}$ & $\begin{array}{l}\stackrel{0}{0} \\
\stackrel{N}{0} \\
\bar{\alpha}\end{array}$ & $\begin{array}{l}\stackrel{2}{2} \\
\stackrel{m}{\alpha} \\
\end{array}$ & $\begin{array}{l}0 \\
\frac{0}{8} \\
\frac{1}{\alpha}\end{array}$ & $\stackrel{\mathscr{L}}{\mathbb{N}}$ & 总 & $\frac{m}{\frac{m}{a}}$ & ริ & $\frac{\mathfrak{T}}{a}$ & 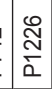 & \begin{tabular}{|l|}
$\overline{\check{D}}$ \\
$\underline{\alpha}$
\end{tabular} & $\mid \begin{array}{c}\tilde{T} \\
\frac{\tilde{J}}{\alpha}\end{array}$ & 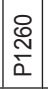 & $\underset{ }{\bar{a}}$ & $\frac{\infty}{i}$ & 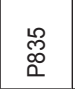 & $\stackrel{0}{i}$ & 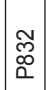 & $\stackrel{\overline{2}}{\alpha}$ & $\begin{array}{l}\tilde{m} \\
\tilde{y} \\
a\end{array}$ & $\hat{\underline{p}}$ & $\frac{\sqrt[b]{o f}}{a}$ \\
\hline 옹 & 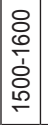 & $\begin{array}{l} \\
\infty \\
\infty \\
\stackrel{\infty}{\circ} \\
\end{array}$ & 오․ & 导 & 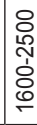 & 윰 & 응 & 㤂 & ০ & : & 守 & ஜ & 怘 & \begin{tabular}{l}
$\stackrel{8}{\circ}$ \\
\hdashline
\end{tabular} & 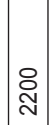 & 怘 & 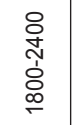 & 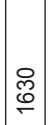 & 怘 & ஜ్రి & 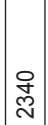 & 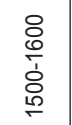 & প্ণু & 足 & ஷి \\
\hline 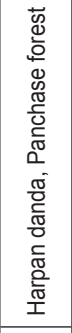 & 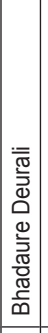 & 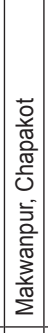 & 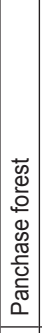 & 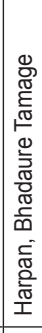 & 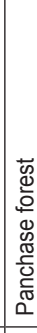 & 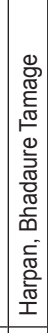 & 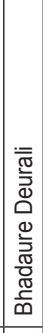 & 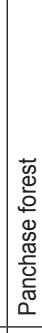 & 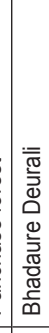 & 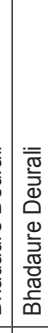 & 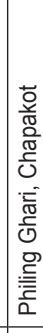 & 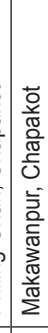 & 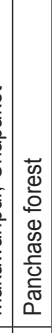 & 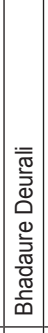 & 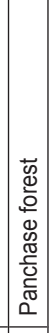 & 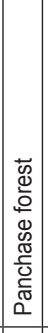 & 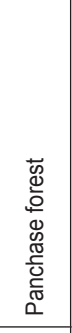 & 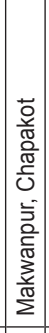 & 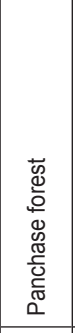 & 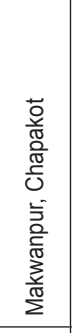 & 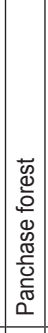 & 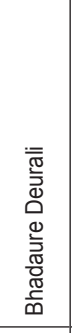 & 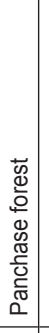 & 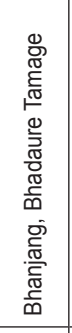 & 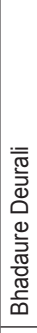 \\
\hline$\frac{\frac{0}{0}}{\frac{0}{1}}$ & \begin{tabular}{|l|}
$\frac{0}{\frac{0}{x}}$ \\
\end{tabular} & 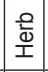 & $\begin{array}{l}\frac{0}{0} \\
\frac{\mathbf{m}}{1}\end{array}$ & $\begin{array}{l}\frac{O}{\bar{O}} \\
\frac{1}{I}\end{array}$ & $\begin{array}{l}\frac{9}{\bar{y}} \\
\frac{\mathrm{O}}{\mathrm{s}}\end{array}$ & $\begin{array}{l}\frac{0}{\overline{0}} \\
\frac{\mathbf{D}}{2}\end{array}$ & $\begin{array}{l}\frac{O}{\bar{O}} \\
\frac{\mathrm{O}}{\mathrm{s}}\end{array}$ & \begin{tabular}{|l}
$\frac{0}{0}$ \\
$\frac{0}{1}$
\end{tabular} & $\frac{\frac{0}{0}}{\frac{0}{1}}$ & $\frac{\frac{0}{0}}{\frac{0}{1}}$ & $\frac{\frac{0}{0}}{\frac{0}{1}}$ & $\frac{\frac{0}{0}}{\frac{0}{1}}$ & $\frac{\frac{0}{0}}{\mathrm{~m}}$ & 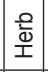 & $\begin{array}{l}\frac{0}{\overline{0}} \\
\frac{\mathbf{D}}{1}\end{array}$ & $\begin{array}{l}\frac{0}{0} \\
\frac{\mathbf{w}}{1}\end{array}$ & $\begin{array}{l}\frac{0}{0} \\
\frac{0}{1}\end{array}$ & $\begin{array}{l}\frac{0}{0} \\
\frac{\mathbf{m}}{1}\end{array}$ & $\frac{\frac{0}{0}}{\frac{0}{1}}$ & $\begin{array}{l}\frac{0}{0} \\
\frac{0}{1}\end{array}$ & $\begin{array}{l}\frac{0}{\overline{0}} \\
\frac{1}{1}\end{array}$ & $\begin{array}{l}\frac{0}{\overline{0}} \\
\frac{1}{1}\end{array}$ & \begin{tabular}{|l|}
$\frac{0}{\overline{0}}$ \\
\end{tabular} & $\begin{array}{l}\frac{0}{\frac{0}{0}} \\
\frac{1}{1}\end{array}$ & $\begin{array}{l}\frac{0}{0} \\
\frac{0}{1}\end{array}$ \\
\hline 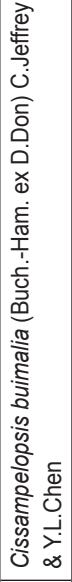 & 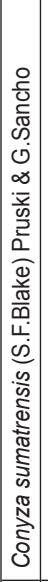 & 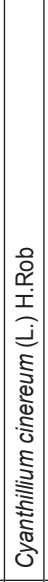 & 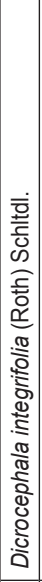 & 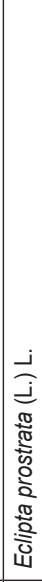 & 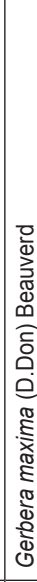 & 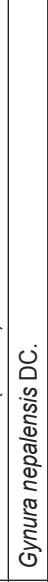 & 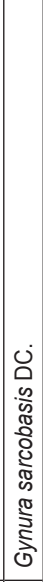 & 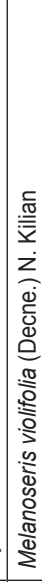 & 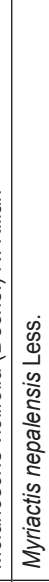 & 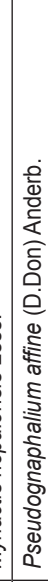 & $\mid$ & 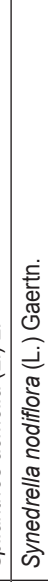 & 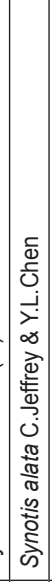 & 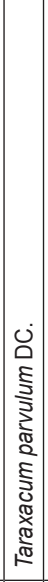 & 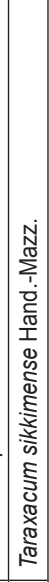 & 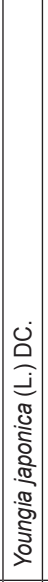 & 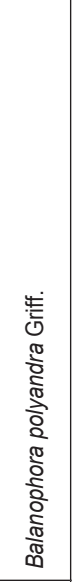 & 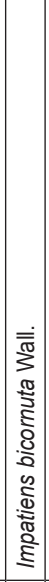 & 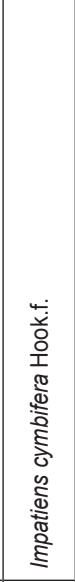 & 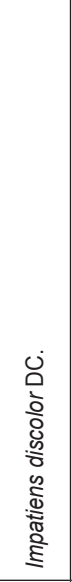 & 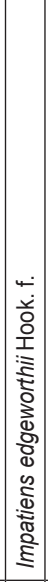 & 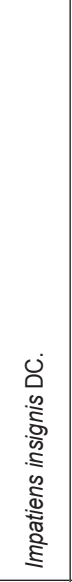 & 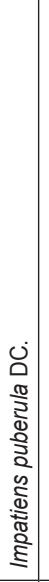 & 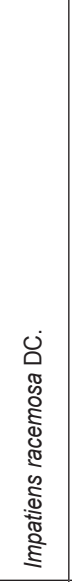 & 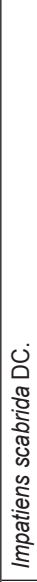 \\
\hline 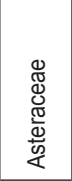 & 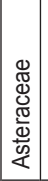 & 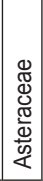 & 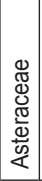 & 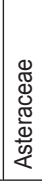 & 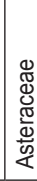 & 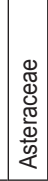 & 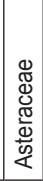 & 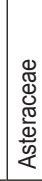 & 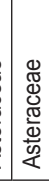 & 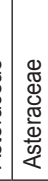 & 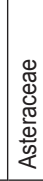 & 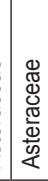 & 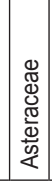 & 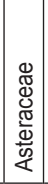 & 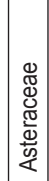 & 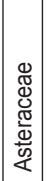 & 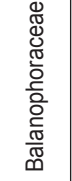 & 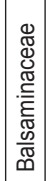 & 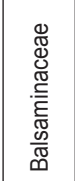 & 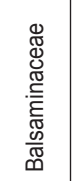 & 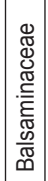 & 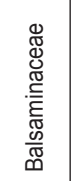 & 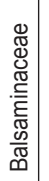 & 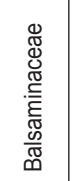 & 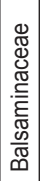 \\
\hline श் & $\infty$ & $\dot{\infty}$ & $\underset{\infty}{\infty}$ & $\infty$ & 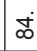 & $\infty$ & 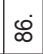 & $\hat{\infty}$ & $\infty$ & $\infty$ & 8 & $\dot{\sigma}$ & ๙่ & கి & $\dot{\sigma}$ & வீ & $\dot{8}$ & $\dot{o}$ & $\stackrel{\infty}{\infty}$ & த் & $\%$ & $\dot{\circ}$ & $\stackrel{ }{~}$ & $\stackrel{\varnothing}{\varnothing}$ & 응 \\
\hline
\end{tabular}




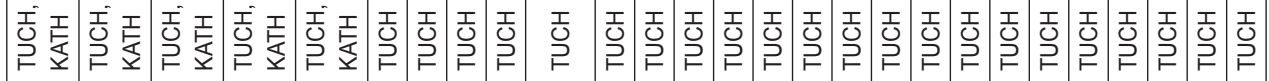

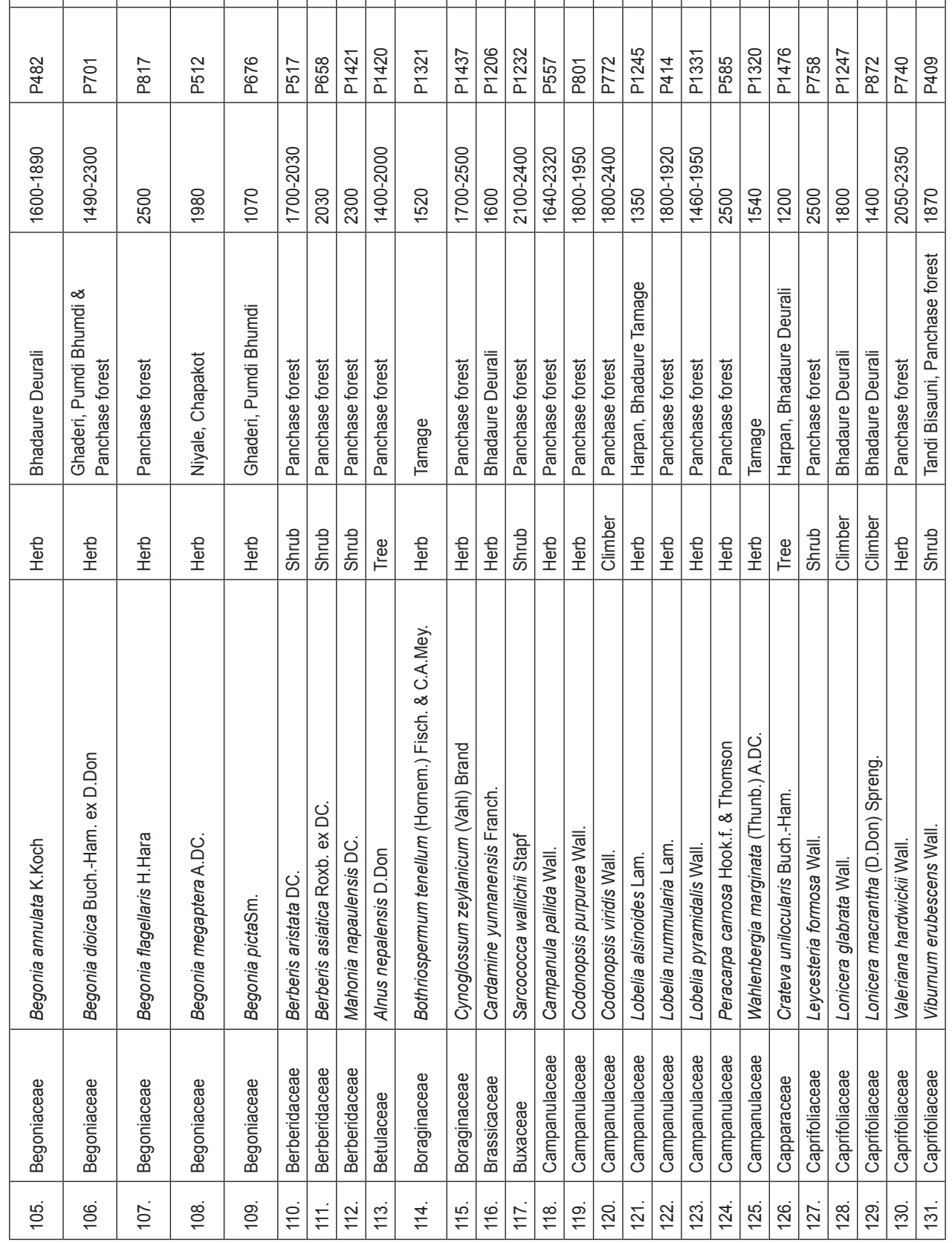




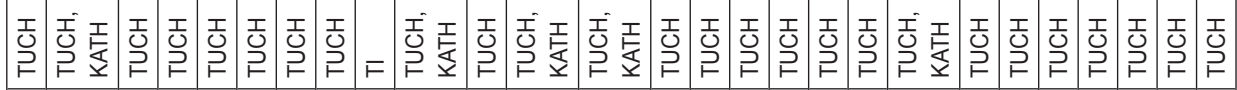

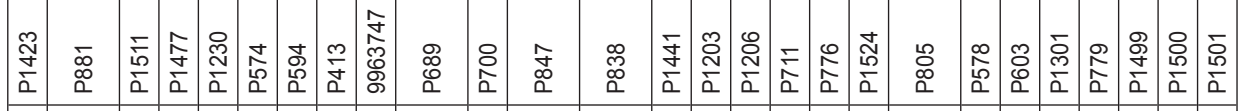

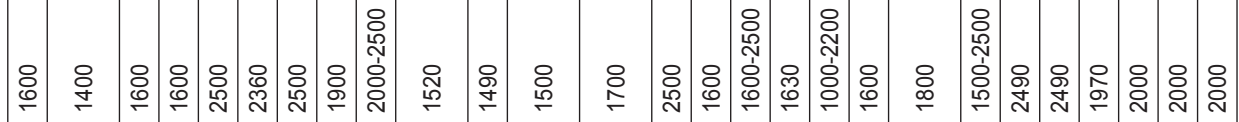

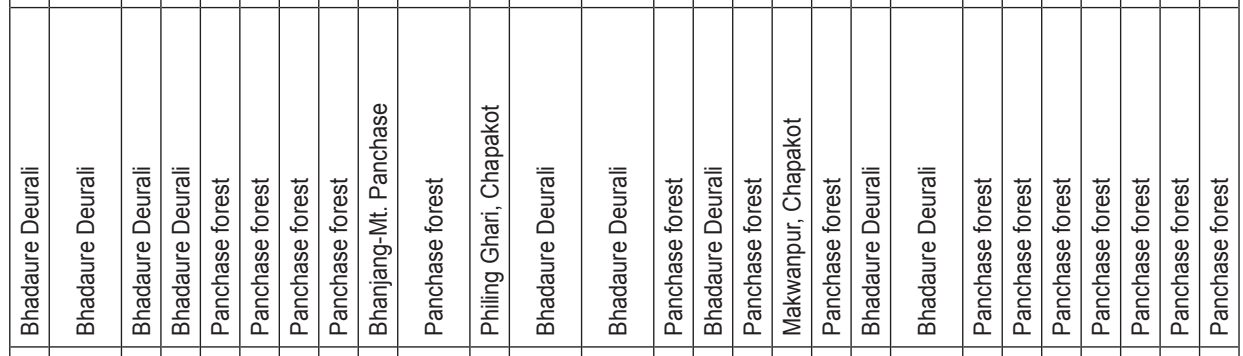

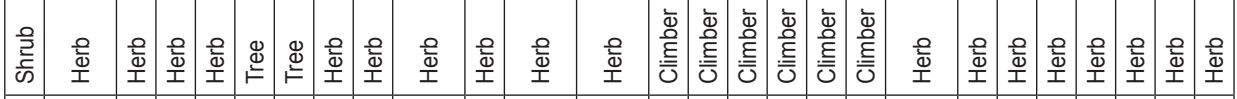

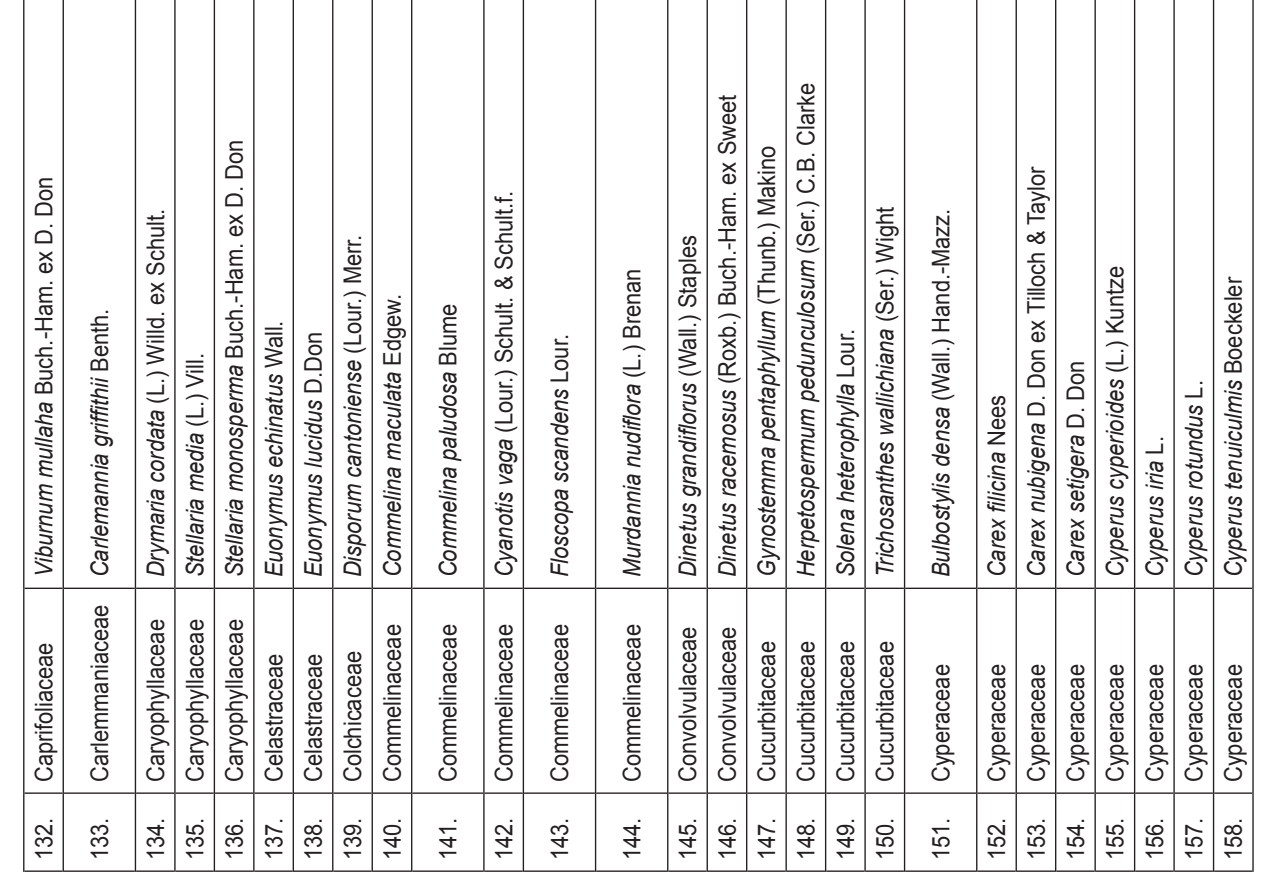




\begin{tabular}{|c|c|c|c|c|c|c|c|c|c|c|c|c|c|c|c|c|c|c|c|c|c|c|c|c|c|c|}
\hline $\begin{array}{l}\text { 든 } \\
\stackrel{2}{P}\end{array}$ & 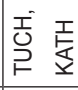 & 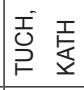 & $\begin{array}{l}\text { I } \\
\text { O } \\
P\end{array}$ & I & $\begin{array}{l}\mathrm{I} \\
\mathrm{U} \\
\mathrm{P}\end{array}$ & $\begin{array}{l}\mathrm{T} \\
\mathrm{U} \\
\mathrm{P}\end{array}$ & $\begin{array}{l}\mathrm{I} \\
\mathrm{U} \\
\longmapsto\end{array}$ & 工 & 壱 & T & 5 & & $\begin{array}{l}\mathrm{I} \\
\mathrm{U} \\
\mathrm{P}\end{array}$ & $\begin{array}{l}\text { 덩 } \\
\stackrel{1}{ }\end{array}$ & $\begin{array}{l}\mathrm{I} \\
\mathrm{I} \\
\mathrm{P}\end{array}$ & $\begin{array}{l}\mathrm{I} \\
\mathrm{I} \\
\mathrm{P} \\
\end{array}$ & $\begin{array}{l}\text { 덩 } \\
\mathrm{U} \\
\longmapsto\end{array}$ & $\begin{array}{l}\mathrm{I} \\
\mathrm{S} \\
\mathrm{P}\end{array}$ & 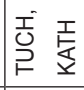 & $\begin{array}{l}\mathrm{T} \\
\mathrm{O} \\
\mathrm{P} \\
\end{array}$ & $\begin{array}{l}\mathrm{T} \\
\mathrm{U} \\
\mathrm{U} \\
\mathrm{P}\end{array}$ & 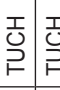 & \begin{tabular}{l|l}
$\mathrm{I}$ & $\mathrm{I}$ \\
$\mathrm{U}$ & $\mathrm{I}$ \\
& $\mathrm{P}$ \\
\end{tabular} & $\begin{array}{l}\mathrm{T} \\
\mathrm{J} \\
\mathrm{P}\end{array}$ & $\begin{array}{l}\text { T } \\
\text { ? } \\
\end{array}$ & $\begin{array}{l}\text { T } \\
\text { U } \\
\text { P }\end{array}$ \\
\hline 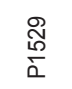 & $\frac{\stackrel{\infty}{\sigma}}{\dot{\alpha}}$ & $\begin{array}{l}\mathbb{\Xi} \\
\propto \\
0\end{array}$ & $\begin{array}{l}\stackrel{8}{8} \\
\text { مी } \\
\text { a }\end{array}$ & $\begin{array}{l}\stackrel{\infty}{\infty} \\
\underset{\infty}{\infty}\end{array}$ & $\begin{array}{l}\infty \\
\propto \\
0\end{array}$ & $\frac{d}{\frac{8}{0}}$ & 卓 & ฉิ & 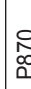 & 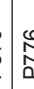 & 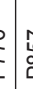 & & 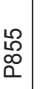 & 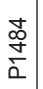 & 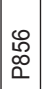 & $\begin{array}{l}\infty \\
\stackrel{\infty}{\alpha} \\
\alpha\end{array}$ & $\begin{array}{l}9 \\
0 \\
0 \\
0\end{array}$ & 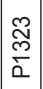 & $\underset{\substack{\infty \\
\infty}}{\mathbb{N}}$ & $\begin{array}{l}0 \\
\stackrel{0}{0} \\
0 \\
0\end{array}$ & ఏ & \begin{tabular}{c|c}
$\overline{0}$ \\
0 \\
0
\end{tabular} & \begin{tabular}{l|l}
$\stackrel{ \pm}{ \pm}$ \\
$\stackrel{2}{\alpha}$
\end{tabular} & $\stackrel{\mathscr{N}}{\mathbb{N}}$ & $\frac{\substack{f \\
2}}{2}$ & 玄 \\
\hline ঃ & 웅 & 옴 & 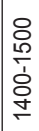 & 各 & $\begin{array}{l}\text { 웅 } \\
\text { ஸे } \\
\text { oे } \\
\text { œ }\end{array}$ & 옹 & 암 & 竎 & g্ & ๕్ & 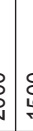 & & 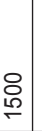 & ঃ্ণ & 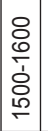 & $\begin{array}{l}\stackrel{0}{2} \\
\stackrel{2}{\sigma}\end{array}$ & 号 & 좀 & 움 & 움 & 完 & 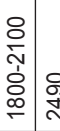 & 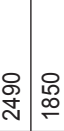 & 온 & 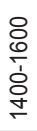 & 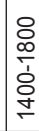 \\
\hline 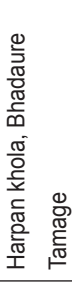 & 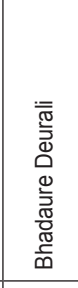 & 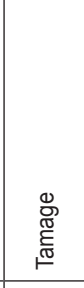 & 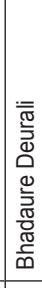 & 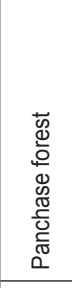 & 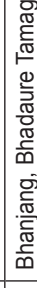 & 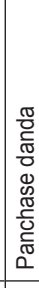 & 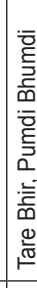 & 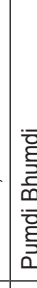 & 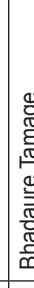 & 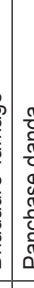 & 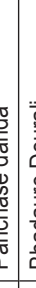 & & 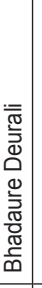 & 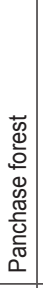 & 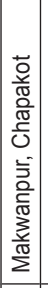 & 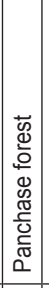 & 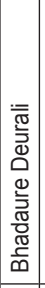 & 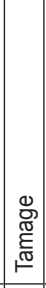 & 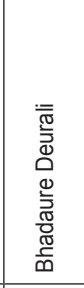 & 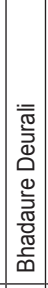 & 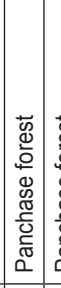 & 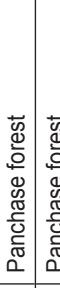 & 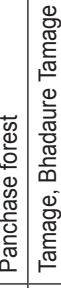 & 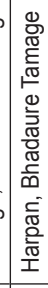 & 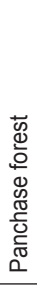 & 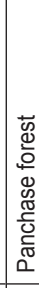 \\
\hline$\stackrel{\Xi}{\stackrel{\Xi}{*}}$ & 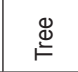 & $\begin{array}{l}\frac{0}{0} \\
\frac{10}{1}\end{array}$ & 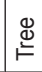 & $\begin{array}{l}\frac{0}{0} \\
\frac{10}{1}\end{array}$ & है & 总 & 忍 & 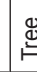 & 旅 & $\frac{d}{d}$ & $\frac{5}{0}$ & & $\frac{\stackrel{\circ}{\bar{\oplus}}}{\underline{1}}$ & $\begin{array}{l}\frac{0}{2} \\
\frac{2}{\omega}\end{array}$ & $\mid$\begin{tabular}{|l}
$\frac{0}{2}$ \\
क \\
क
\end{tabular} & $\mid \begin{array}{l} \\
\frac{2}{2} \\
\text { क } \\
\text { क }\end{array}$ & $\begin{array}{l}\frac{0}{0} \\
\frac{\mathrm{\omega}}{\mathrm{T}}\end{array}$ & 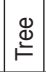 & $\begin{array}{l}\frac{0}{0} \\
\frac{1}{1}\end{array}$ & 䱛 & $\begin{array}{ll}\frac{0}{2} \\
\frac{2}{5} \\
\text { क }\end{array}$ & \begin{tabular}{l|l}
$\frac{0}{\frac{0}{9}}$ & $\frac{9}{9}$ \\
$\frac{9}{1}$
\end{tabular} & \begin{tabular}{l|l}
$\frac{0}{0}$ \\
$\frac{1}{1}$
\end{tabular} & $\frac{}{\frac{0}{1}}$ & 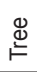 & 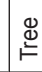 \\
\hline 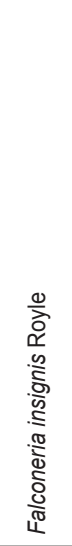 & 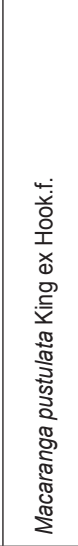 & 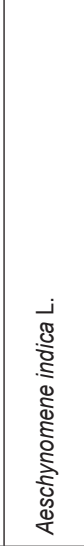 & 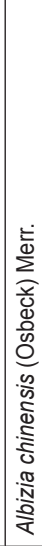 & 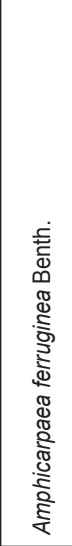 & 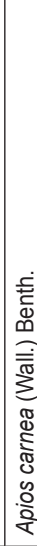 & 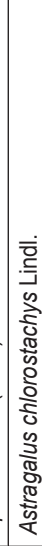 & 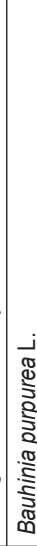 & 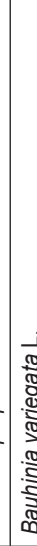 & 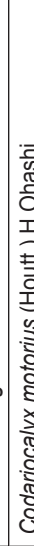 & 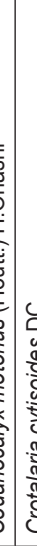 & 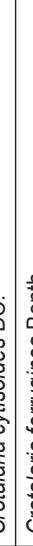 & 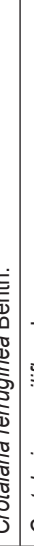 & 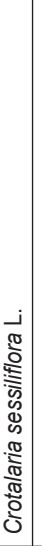 & 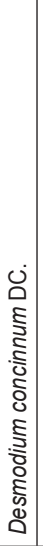 & 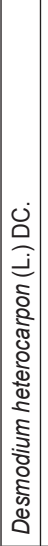 & 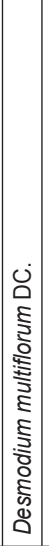 & 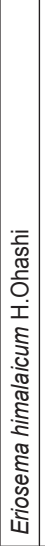 & 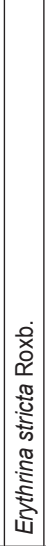 & 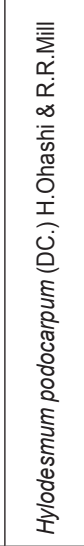 & 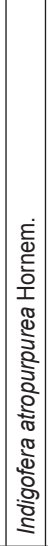 & 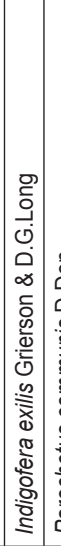 & 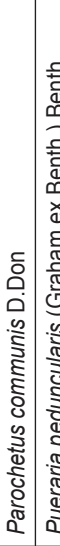 & 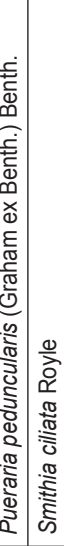 & 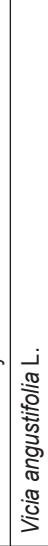 & 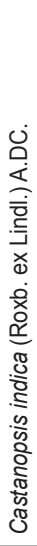 & 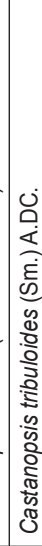 \\
\hline 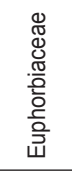 & 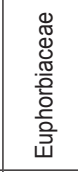 & 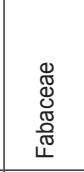 & 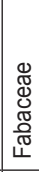 & 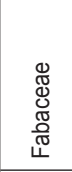 & $\begin{array}{l}\mathbb{\Xi} \\
\mathbb{\Xi} \\
\mathbb{\pi} \\
\mathbb{\sigma}\end{array}$ & 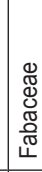 & 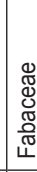 & 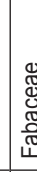 & \begin{tabular}{|l}
$\pi$ \\
$d$ \\
$d$ \\
$\pi$ \\
$\pi$ \\
$\pi$
\end{tabular} & & & & 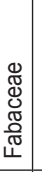 & $\begin{array}{l}\mathbb{\Xi} \\
\mathbb{\Xi} \\
\mathbb{\Xi} \\
\mathbb{J} \\
\mathbb{\widetilde { J }} \\
\leftarrow\end{array}$ & 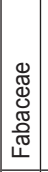 & 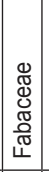 & 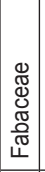 & 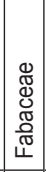 & 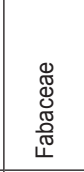 & 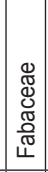 & 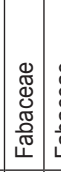 & 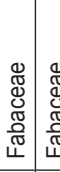 & 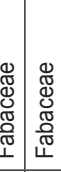 & 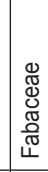 & 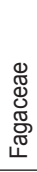 & 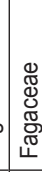 \\
\hline$\stackrel{\infty}{\infty}$ & 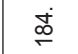 & 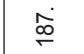 & $\stackrel{\infty}{\sim}$ & $\stackrel{\stackrel{\infty}{\circ}}{\circ}$ & $\stackrel{\circ}{-}$ & \% & 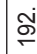 & & & & & & $\stackrel{\mathscr{\sigma}}{\leftarrow}$ & के & $\stackrel{\infty}{\circ}$ & $\stackrel{\text { I }}{\circ}$ & ஓి & i্. & ๙ิ่ & ¿ें & 兑 & ֻ̊ำ & 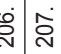 & 家 & $\stackrel{\circ}{\sim}$ & $\dot{\check{N}}$ \\
\hline
\end{tabular}




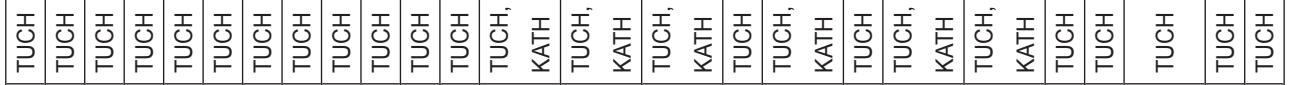

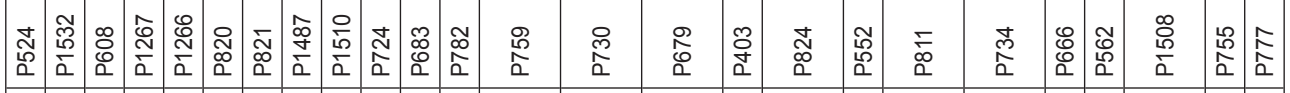

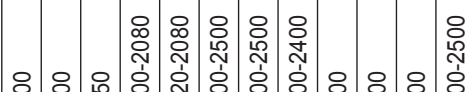

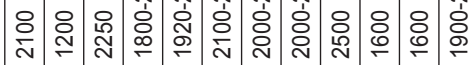

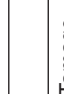

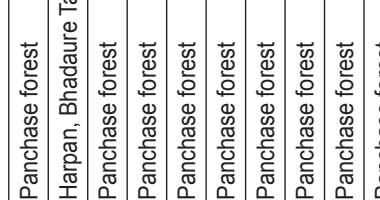

言

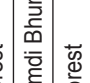

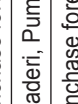

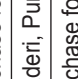

$\frac{\pi}{\frac{\pi}{0}}$

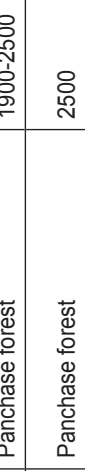

$\operatorname{lin}^{3}$

递

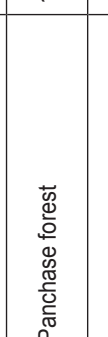

옹 응

율

กั่

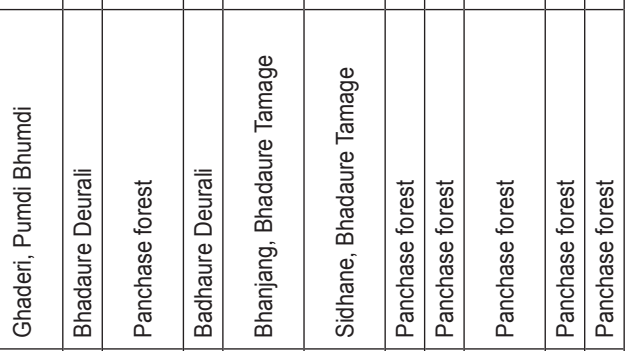

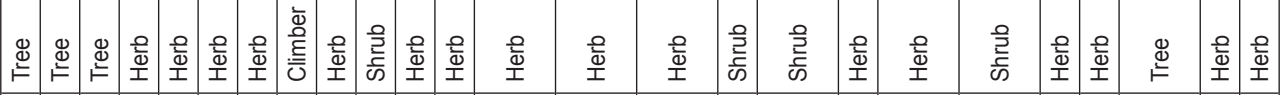

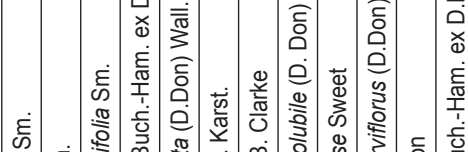

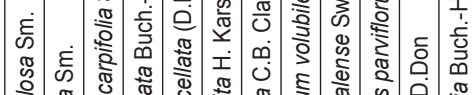

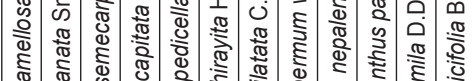

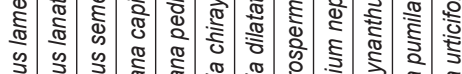

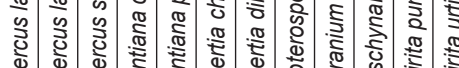

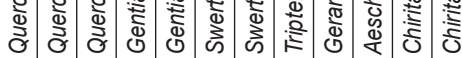

(

\begin{tabular}{|c|c|c|c|c|c|c|c|c|c|c|c|c|c|c|c|c|c|c|c|c|}
\hline 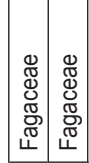 & 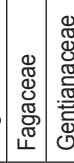 & & 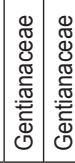 & 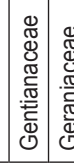 & 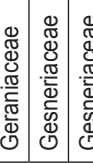 & 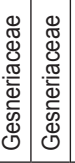 & 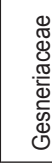 & & 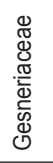 & 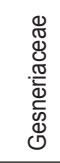 & 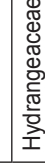 & & & & 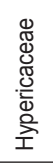 & 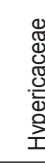 & & & & \\
\hline$\stackrel{\rho}{\rightleftharpoons}$ & $\stackrel{\stackrel{\sim}{N}}{\stackrel{n}{N}}$ & & $\stackrel{\circ}{\circ}$ & $\frac{\dot{\Omega}}{\mathrm{N}} \underset{\text { (2) }}{ }$ & ลิ & ลี่ & & & & ¿্ं & & & & & ळ్ & & & & & 岕 \\
\hline
\end{tabular}


Bhandari et al.: A checklist of flowering plants of Panchase.

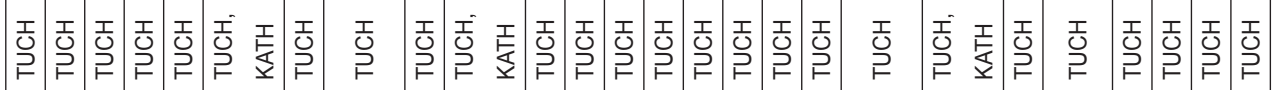

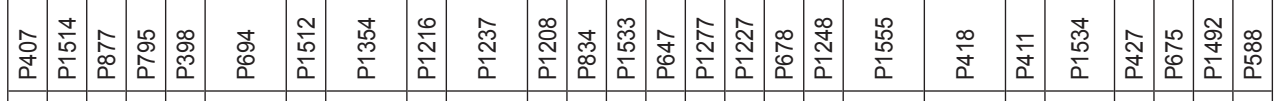

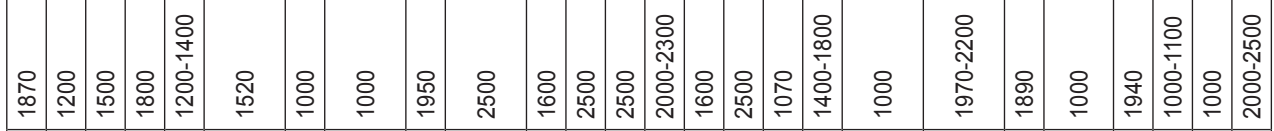

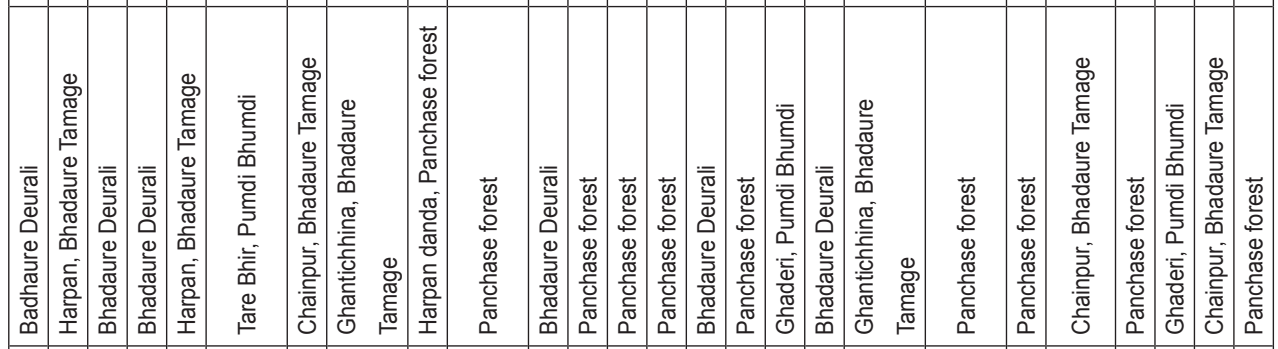

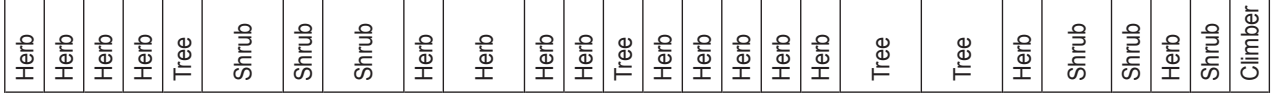

\begin{tabular}{|c|c|c|c|c|c|c|c|c|c|c|c|c|c|c|c|c|c|c|c|c|c|}
\hline 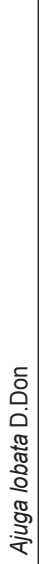 & 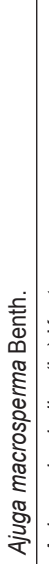 & 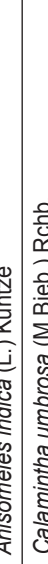 & 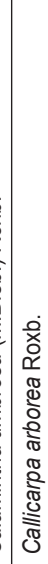 & 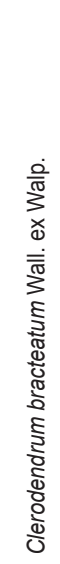 & 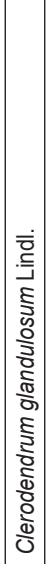 & 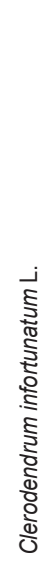 & 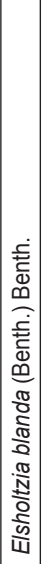 & 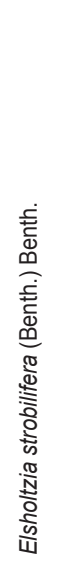 & 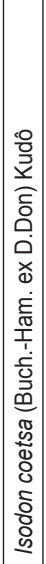 & 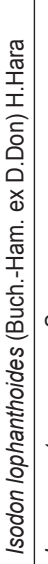 & 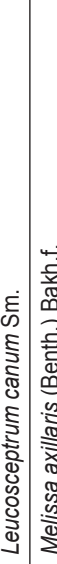 & 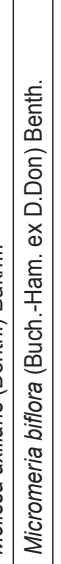 & 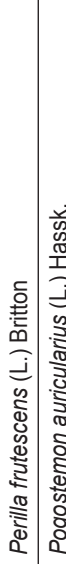 & 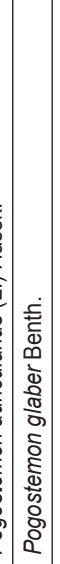 & 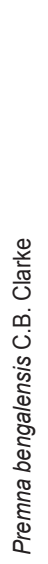 & 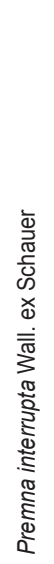 & 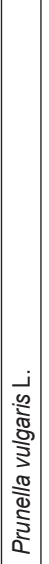 & 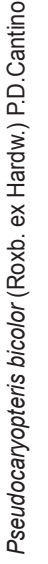 & 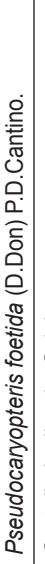 & 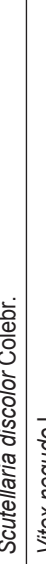 & 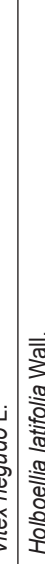 \\
\hline 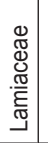 & 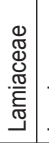 & 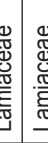 & 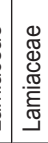 & 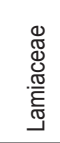 & 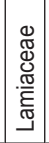 & 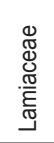 & 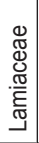 & 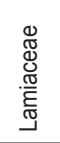 & 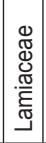 & 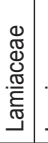 & 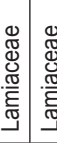 & 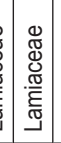 & 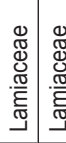 & 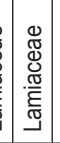 & 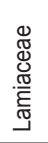 & 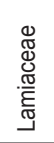 & 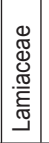 & 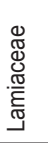 & $\begin{array}{l}\mathbb{\Perp} \\
\mathbb{\pm} \\
. \\
. \\
\end{array}$ & 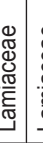 & \\
\hline$\hat{\tilde{N}}$ & $\stackrel{\infty}{\sim}$ & 鹿 & & $\dot{d}$ & $\stackrel{\mathcal{i}}{\mathrm{N}}$ & $\stackrel{\stackrel{d}{\sim}}{\sim}$ & & $\stackrel{\text { I }}{\text { d }}$ & 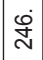 & $\stackrel{\dot{d}}{\mathbf{N}}$ & 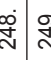 & 灾 & 灾 స్ట & $\stackrel{\text { }}{\stackrel{\leftrightarrow}{n}}$ & $\dot{\mathrm{O}}$ & 岕 & 岕 & 岕 & & & \\
\hline
\end{tabular}




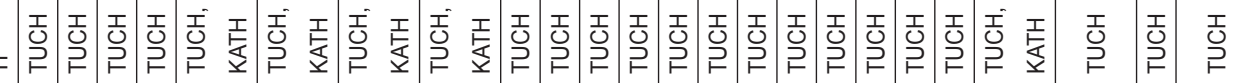

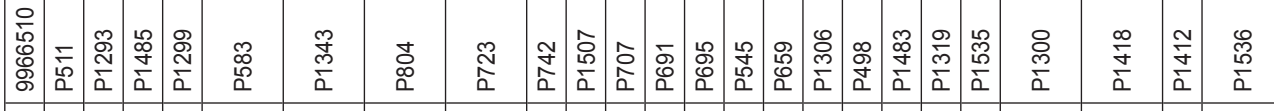

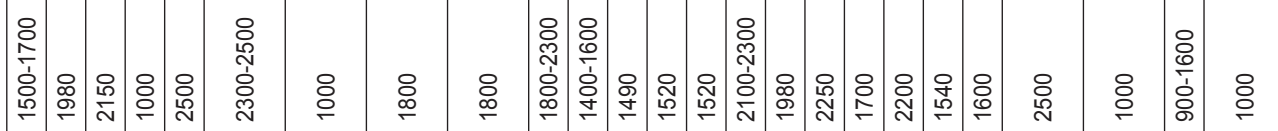

\begin{tabular}{|c|c|c|c|c|c|c|c|c|c|c|c|c|c|c|c|c|c|c|c|c|c|c|c|}
\hline 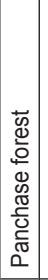 & 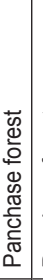 & 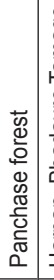 & 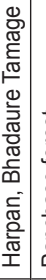 & 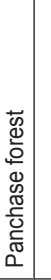 & 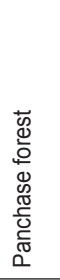 & 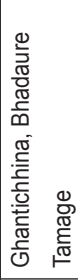 & 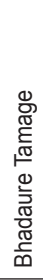 & 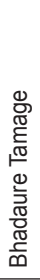 & 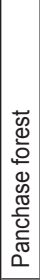 & 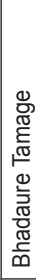 & 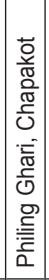 & 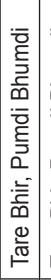 & 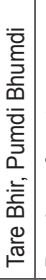 & 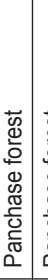 & 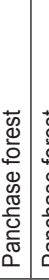 & 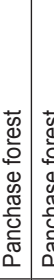 & 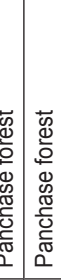 & 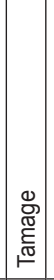 & 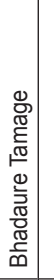 & 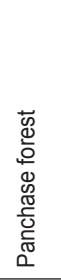 & 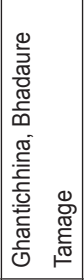 & 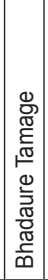 & 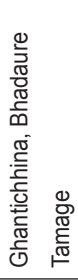 \\
\hline 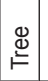 & 离 & \begin{tabular}{l|l}
$\stackrel{\Xi}{\mathbb{E}}$ \\
$\stackrel{2}{*}$
\end{tabular} & $\underset{\mathbb{\Phi}}{\stackrel{\Phi}{\sharp}}$ & 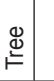 & 导 & 离 & 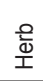 & $\frac{\text { 웜 }}{\underline{\underline{\Phi}}}$ & $\begin{array}{l}\frac{0}{\frac{0}{1}} \\
\frac{1}{1}\end{array}$ & \begin{tabular}{|l}
$\frac{0}{0}$ \\
$\frac{0}{1}$
\end{tabular} & \begin{tabular}{|l|}
$\frac{0}{0}$ \\
$\frac{0}{1}$
\end{tabular} & 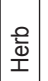 & $\frac{\stackrel{0}{\bar{\omega}}}{\frac{1}{I}}$ & 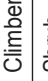 & $\frac{2}{2}$ & 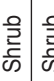 & 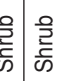 & 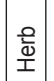 & 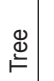 & 导 & $\begin{array}{l}\text { 롤 } \\
\text { 竞 }\end{array}$ & $\frac{\frac{0}{0}}{\frac{0}{1}}$ & $\stackrel{\bigotimes}{\stackrel{\Xi}{\models}}$ \\
\hline 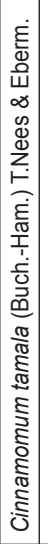 & 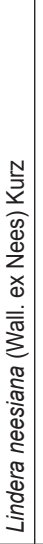 & 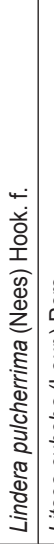 & 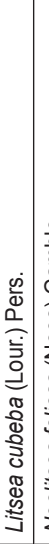 & 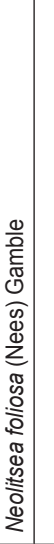 & 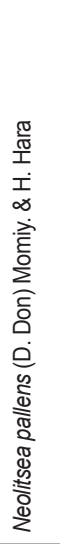 & 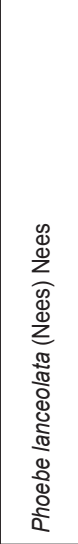 & 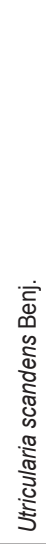 & 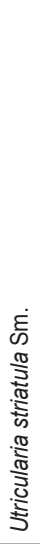 & 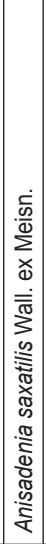 & 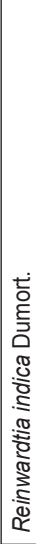 & 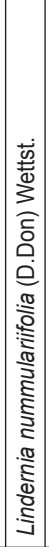 & 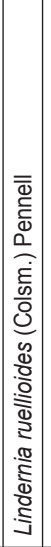 & 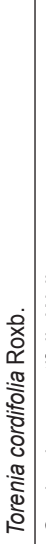 & 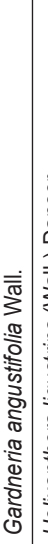 & 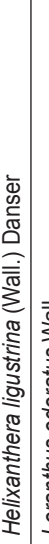 & 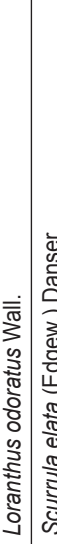 & 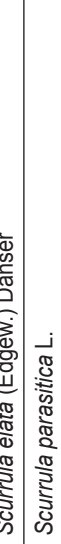 & 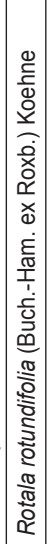 & 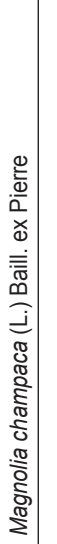 & 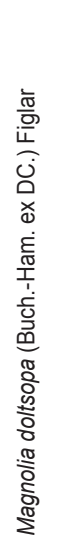 & 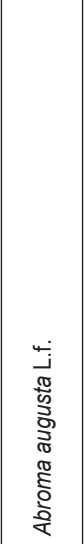 & 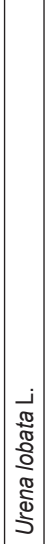 & 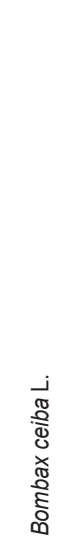 \\
\hline 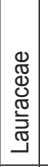 & 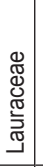 & 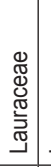 & 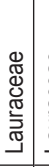 & 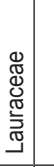 & 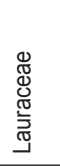 & 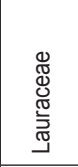 & 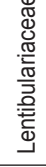 & 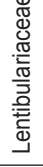 & 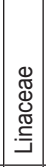 & 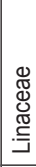 & 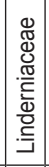 & 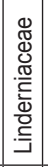 & 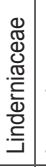 & 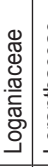 & 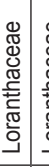 & 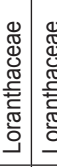 & 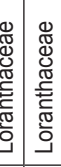 & 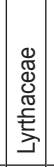 & 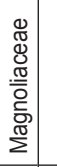 & 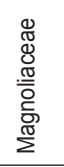 & $\begin{array}{l}\mathbb{\Xi} \\
\mathbb{\Xi} \\
\mathbb{J} \\
\mathbb{\pi} \\
\stackrel{2}{\pi} \\
\sum\end{array}$ & 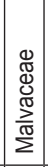 & 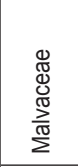 \\
\hline 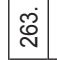 & 志 & 岕 & $\stackrel{c}{\circledR}$ & 灾 & $\stackrel{\infty}{\stackrel{0}{\circ}}$ & @ి & సิ & $\hat{\sim}$ & $\stackrel{N}{N}$ & $\hat{\sim}$ & $\stackrel{\mathbb{N}}{N}$ & 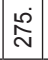 & 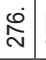 & $\hat{\tilde{N}}$ & $\stackrel{\infty}{\stackrel{\infty}{\sim}}$ & 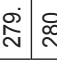 & 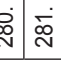 & 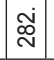 & $\stackrel{\infty}{\sim}$ & 品 & 吕 & $\begin{array}{l}\dot{0} \\
\stackrel{0}{ }\end{array}$ & 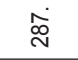 \\
\hline
\end{tabular}


Bhandari et al.: A checklist of flowering plants of Panchase.

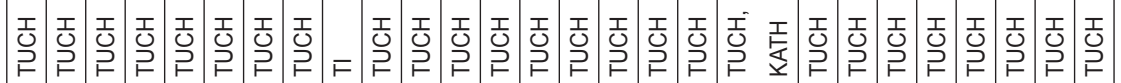

章

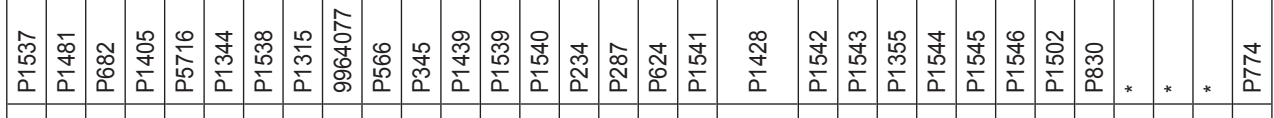

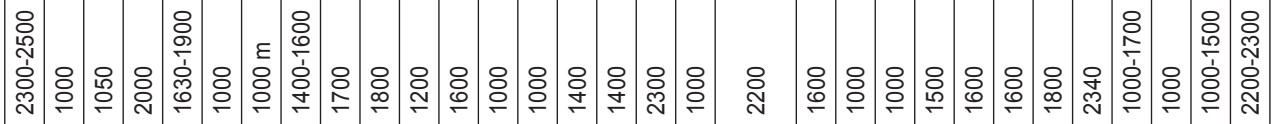

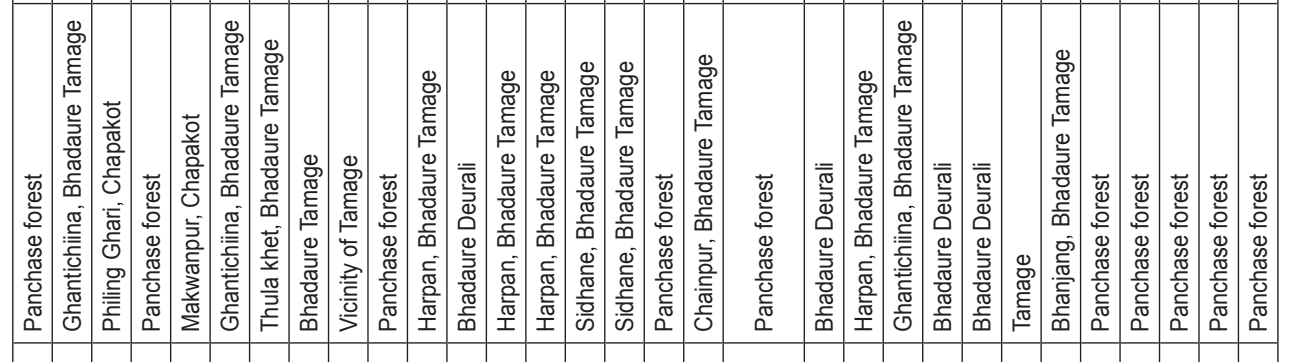

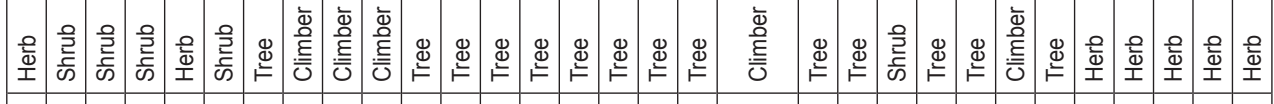

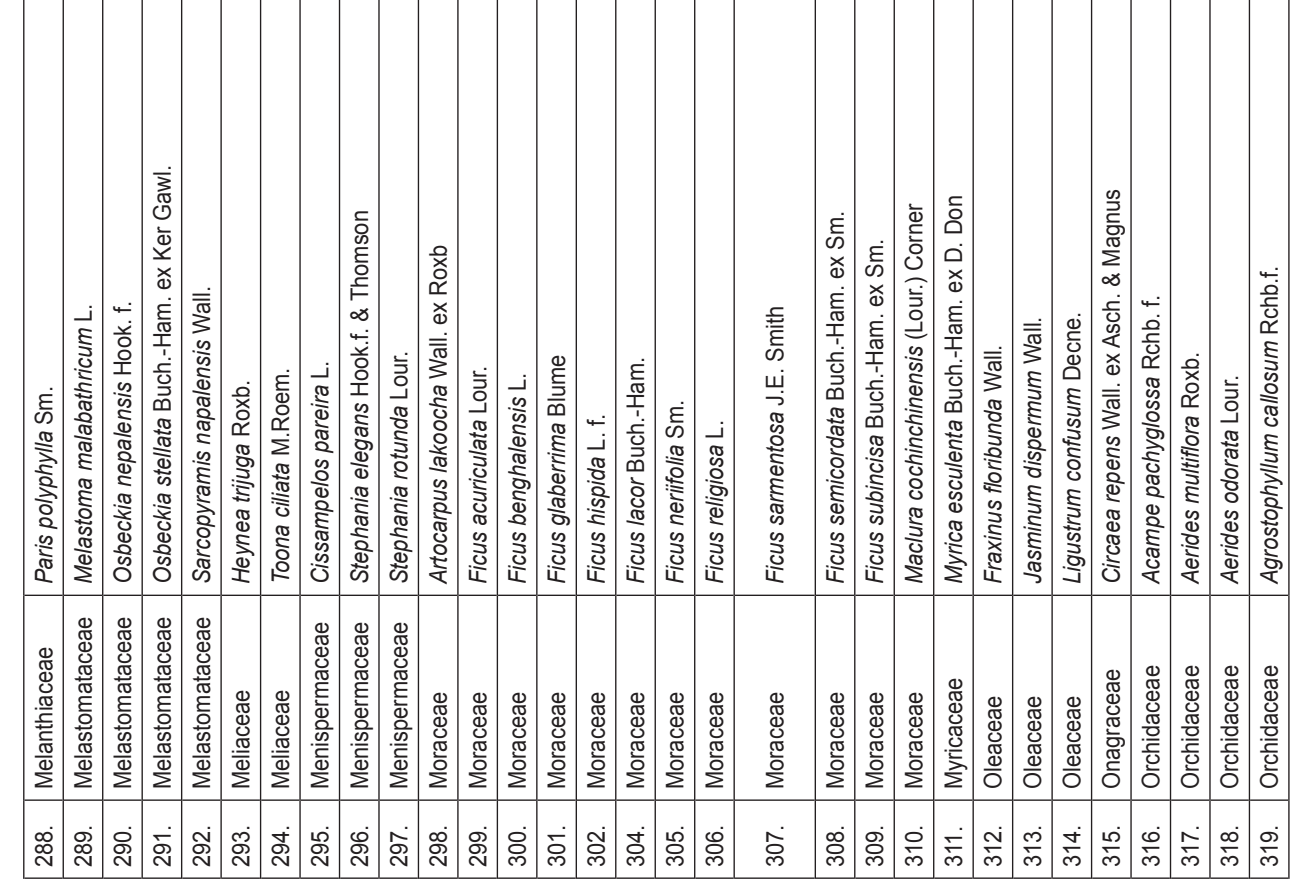




\begin{tabular}{|c|c|c|c|c|c|c|c|c|c|c|c|c|c|c|c|c|c|c|c|c|c|c|c|c|c|c|}
\hline 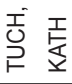 & & \begin{tabular}{|l|} 
\\
\\
\\
\\
\end{tabular} & 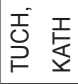 & & $\begin{array}{l}\text { T } \\
\text { O } \\
\end{array}$ & & $\begin{array}{l}\text { I } \\
\stackrel{\mathrm{U}}{\mathrm{r}}\end{array}$ & & & & & 건 & II & & \begin{tabular}{|l} 
I \\
O \\
$?$
\end{tabular} & & 焉 & \begin{tabular}{|l} 
\\
\\
\\
\end{tabular} & $\begin{array}{ll}\mathrm{I} \\
\mathrm{J}\end{array}$ & $\begin{array}{l}\mathrm{I} \\
\mathrm{U} \\
\mathrm{O}\end{array}$ & & & 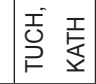 & & & $\begin{array}{l}\mathrm{T} \\
\mathrm{U} \\
\mathrm{P}\end{array}$ \\
\hline $\begin{array}{l}\text { \& } \\
\text { ¿ }\end{array}$ & * & 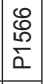 & $\frac{\stackrel{6}{g}}{\square}$ & * & 柋 & * & $\begin{array}{l}\hat{0} \\
\text { in } \\
0\end{array}$ & * & స్ & * & * & $\stackrel{0}{\stackrel{N}{\Sigma}}$ & ஓ্ণ & * & * & & $\underset{\mathbf{S}}{\text { 衣 }}$ & 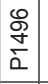 & 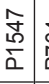 & 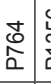 & 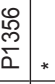 & * & $\begin{array}{l}\stackrel{\infty}{D} \\
\stackrel{D}{\Lambda}\end{array}$ & * & . & $\begin{array}{l}\hat{y} \\
\dot{z} \\
\end{array}$ \\
\hline $\begin{array}{l}\frac{8}{\circ} \\
\frac{1}{8} \\
\frac{0}{0}\end{array}$ & & ঃ & ঃి & & 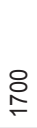 & & ஓ & & 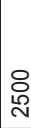 & & & ¿্口 & 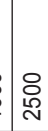 & & & & $\stackrel{\circ}{i}$ & ০্ণ & 怘 & $\stackrel{O}{d}$ & & 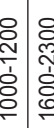 & ঃ্ণ & & & 음 \\
\hline 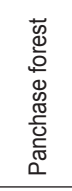 & 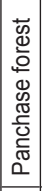 & 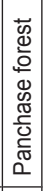 & 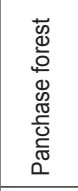 & 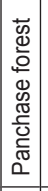 & 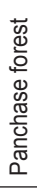 & 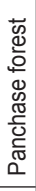 & 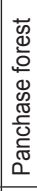 & 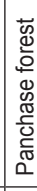 & 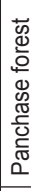 & 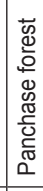 & 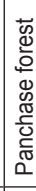 & 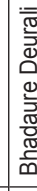 & 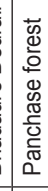 & 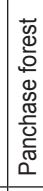 & 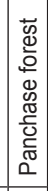 & 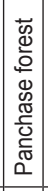 & 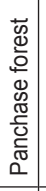 & 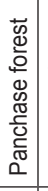 & 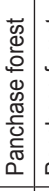 & 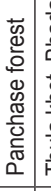 & 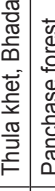 & 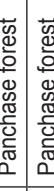 & 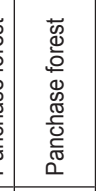 & 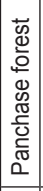 & 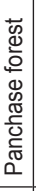 & 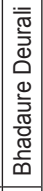 \\
\hline $\begin{array}{l}\text { 迋 } \\
\text { I }\end{array}$ & 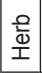 & \begin{tabular}{|l}
$\frac{0}{\overline{0}}$ \\
I
\end{tabular} & $\begin{array}{l}\text { 迋 } \\
\text { I }\end{array}$ & $\begin{array}{l}\frac{0}{\overline{0}} \\
\frac{1}{I}\end{array}$ & $\begin{array}{l}\frac{0}{0} \\
\frac{0}{1}\end{array}$ & $\frac{}{\frac{0}{1}}$ & $\begin{array}{l}\frac{0}{\overline{0}} \\
\frac{0}{1}\end{array}$ & \begin{tabular}{|l}
$\frac{9}{0}$ \\
$\frac{0}{1}$
\end{tabular} & 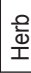 & $\begin{array}{l}\frac{0}{\mathrm{D}} \\
\frac{\mathrm{w}}{\mathrm{s}}\end{array}$ & \begin{tabular}{|l}
$\frac{0}{\overline{0}}$ \\
$\frac{\mathrm{O}}{\mathrm{I}}$
\end{tabular} & $\frac{\frac{0}{\bar{d}}}{\frac{1}{1}}$ & $\frac{\frac{0}{0}}{\mathrm{~d}}$ & $\begin{array}{l}\frac{0}{\bar{\omega}} \\
\frac{1}{1}\end{array}$ & \begin{tabular}{|l}
$\frac{0}{\frac{0}{T}}$ \\
$\frac{1}{1}$
\end{tabular} & $\begin{array}{l}\frac{0}{\overline{0}} \\
\frac{\mathrm{\omega}}{\mathrm{T}}\end{array}$ & 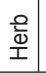 & $\mid \begin{array}{l}\frac{0}{\overline{0}} \\
\frac{1}{1}\end{array}$ & $\frac{\frac{0}{0}}{\frac{0}{1}}$ & $\frac{0}{\frac{0}{0}}$ & $\begin{array}{ll}\frac{0}{9} \\
\frac{9}{9}\end{array}$ & 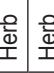 & $\begin{array}{l}\frac{0}{\frac{0}{1}} \\
\frac{1}{1}\end{array}$ & $\frac{0}{\frac{0}{\Phi}}$ & $\frac{\stackrel{0}{\bar{\Phi}}}{\underline{\underline{T}}}$ & \begin{tabular}{|l|}
$\frac{0}{\bar{\theta}}$ \\
$\frac{3}{1}$
\end{tabular} \\
\hline 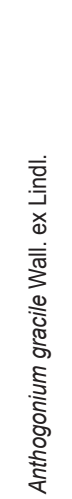 & 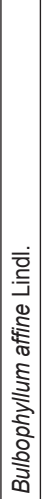 & 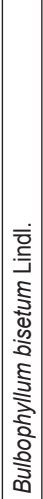 & 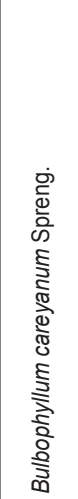 & 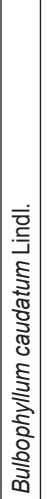 & 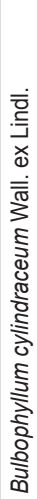 & 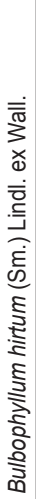 & 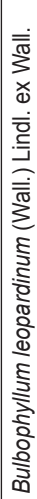 & 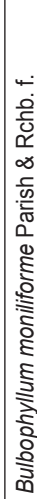 & 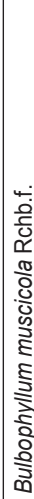 & 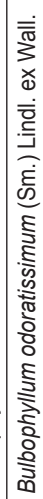 & 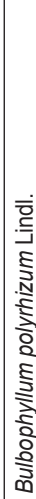 & 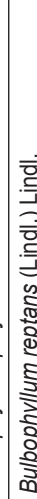 & 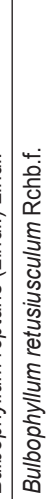 & 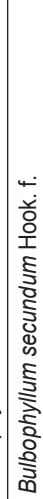 & 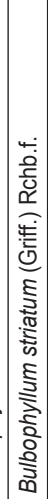 & 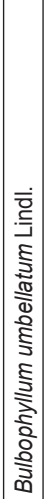 & 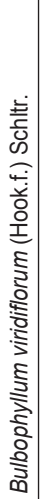 & 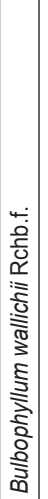 & 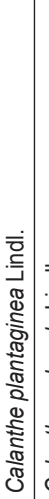 & 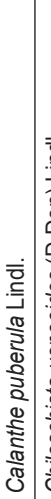 & 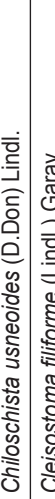 & 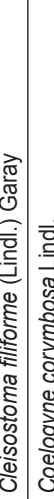 & 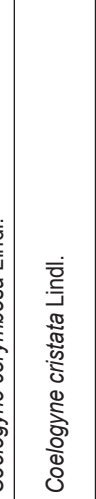 & 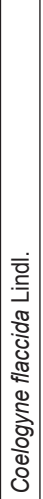 & 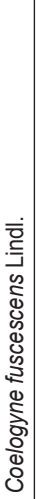 & 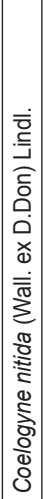 \\
\hline 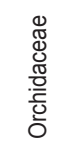 & $\begin{array}{l}\mathbb{\Phi} \\
\mathbb{J} \\
\mathbb{E} \\
\frac{\pi}{0} \\
\frac{\bar{c}}{0} \\
0\end{array}$ & 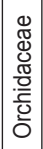 & 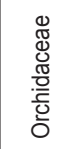 & $\begin{array}{l}\mathbb{8} \\
\mathbb{J} \\
\mathbb{0} \\
\frac{\pi}{0} \\
\bar{c} \\
0 \\
0\end{array}$ & 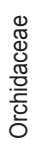 & 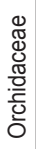 & 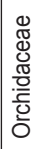 & 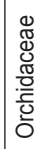 & 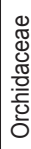 & 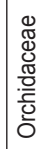 & 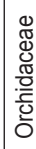 & 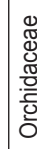 & 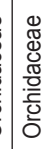 & 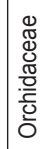 & 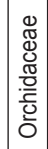 & 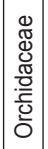 & $\begin{array}{l}\mathbb{\Xi} \\
\mathbb{\Xi} \\
\mathbb{\Xi} \\
\frac{\pi}{0} \\
\frac{\bar{c}}{0} \\
0\end{array}$ & \begin{tabular}{|l|} 
\\
$\mathbb{8}$ \\
$\mathbb{0}$ \\
$\frac{\pi}{0}$ \\
$\frac{0}{0}$ \\
0 \\
\end{tabular} & 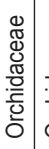 & 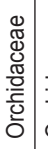 & 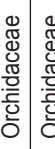 & 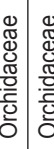 & 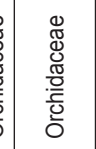 & 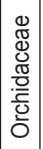 & & 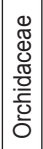 \\
\hline ণ্লি & $\underset{\bar{m}}{\dot{m}}$ & స్ల & ల్లై & 离 & 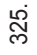 & ঙ্লি & ल & 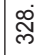 & & m & ळ্ল & 心్ల & 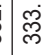 & 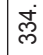 & 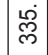 & $\begin{array}{l}\dot{e} \\
\text { m }\end{array}$ & 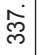 & 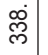 & ల్ల & 守 & 它 & fે & 胥 & 官 & & 它 \\
\hline
\end{tabular}


Bhandari et al:: A checklist of flowering plants of Panchase.

\begin{tabular}{|c|c|c|c|c|c|c|c|c|c|c|c|c|c|c|c|c|c|c|c|c|c|c|c|c|}
\hline 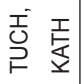 & $\begin{array}{ll}\text { I⿱ } \\
\text { 焉 }\end{array}$ & 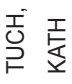 & 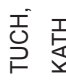 & & $\stackrel{I}{\mathrm{I}}$ & T & $\begin{array}{l}\text { T } \\
\text { C } \\
\text { F }\end{array}$ & $\frac{1}{\mathrm{O}}$ & $\frac{T}{C}$ & $\begin{array}{l}\text { I } \\
\text { 을 } \\
P\end{array}$ & & & & $\begin{array}{l}\text { I } \\
\mathrm{U} \\
\longmapsto\end{array}$ & 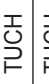 & 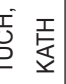 & 돈 & & & \begin{tabular}{ll} 
İ & \multicolumn{1}{c}{} \\
P & 多
\end{tabular} & & & & $\begin{array}{l}\mathrm{I} \\
\mathrm{S} \\
\end{array}$ \\
\hline $\begin{array}{l}\text { Oे } \\
\text { o. }\end{array}$ & $\begin{array}{l}\text { 芯 } \\
\text { 足 }\end{array}$ & $\begin{array}{l}0 \\
\infty \\
0 \\
0\end{array}$ & 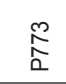 & * & 通 & ลิ & ì & क & 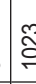 & 总 & * & * & * & 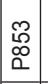 & 量 & 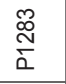 & 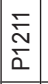 & *. & * & $\begin{array}{l}\stackrel{8}{\circ} \\
\text { م. }\end{array}$ & * & * & * & 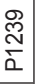 \\
\hline 음 & 吕 & 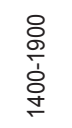 & 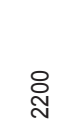 & $\begin{array}{l}\frac{8}{\circ} \\
\text { 홍 }\end{array}$ & ঃ & $\stackrel{\circ}{n}$ & 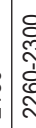 & ঃু & ‡ & 옹 & & & & 各 & & $\stackrel{\circ}{\circ}$ & 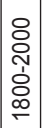 & & & 怘 & & & 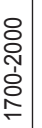 & $\underset{\stackrel{\mathscr{O}}{\circ}}{\stackrel{\circ}{\circ}}$ \\
\hline 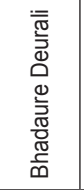 & 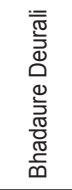 & 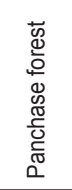 & 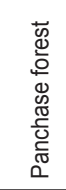 & 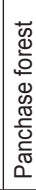 & 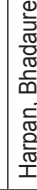 & \begin{tabular}{l}
\multicolumn{1}{c}{} \\
0 \\
0 \\
0 \\
0 \\
0 \\
0 \\
0 \\
0 \\
0 \\
0 \\
0 \\
0
\end{tabular} & 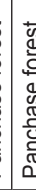 & 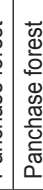 & 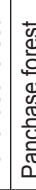 & 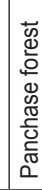 & 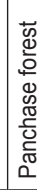 & 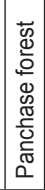 & 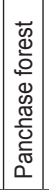 & 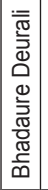 & 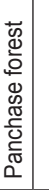 & 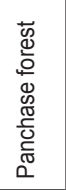 & 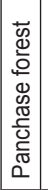 & 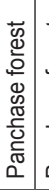 & 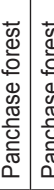 & 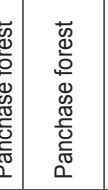 & 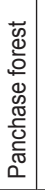 & 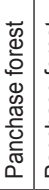 & 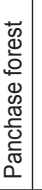 & 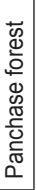 \\
\hline $\begin{array}{l}\frac{0}{0} \\
\frac{0}{1}\end{array}$ & $\begin{array}{l}\frac{0}{\frac{0}{1}} \\
\frac{1}{1}\end{array}$ & $\begin{array}{l}\frac{0}{0} \\
\frac{0}{1}\end{array}$ & $\begin{array}{l}\frac{0}{0} \\
\frac{0}{1}\end{array}$ & $\begin{array}{l}\frac{0}{\mathrm{~d}} \\
\frac{1}{1}\end{array}$ & \begin{tabular}{|l}
$\frac{0}{\overline{0}}$ \\
$\frac{1}{1}$
\end{tabular} & $\frac{O}{\frac{0}{I}}$ & 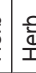 & $\frac{2}{\frac{0}{4}}$ & $\frac{9}{\frac{a}{9}}$ & $\frac{\frac{9}{0}}{\frac{0}{1}}$ & $\begin{array}{l}\frac{9}{\overline{0}} \\
\frac{1}{1}\end{array}$ & 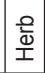 & $\mid \begin{array}{l}\frac{0}{0} \\
\frac{\mathbf{m}}{1}\end{array}$ & $\mid \begin{array}{l}\frac{0}{\Phi} \\
\frac{\bar{\omega}}{1}\end{array}$ & $\frac{\underline{0}}{\underline{\underline{\omega}}}$ & $\begin{array}{l}\frac{0}{0} \\
\frac{0}{1}\end{array}$ & $\mid \begin{array}{l}\frac{0}{\Phi} \\
\frac{\bar{\omega}}{1}\end{array}$ & 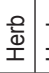 & $\begin{array}{ll}\frac{0}{9} \\
\frac{1}{1} \\
\end{array}$ & $\begin{array}{l}\frac{0}{\frac{0}{0}} \\
\frac{1}{1}\end{array}$ & $\frac{\frac{0}{0}}{\frac{0}{1}}$ & $\frac{0}{\frac{0}{1}}$ & $\begin{array}{l}\frac{0}{\overline{0}} \\
\frac{\mathrm{o}}{1}\end{array}$ & $\frac{\stackrel{0}{\overline{0}}}{\underline{\underline{\Phi}}}$ \\
\hline 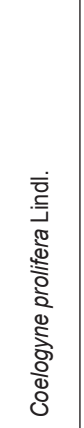 & 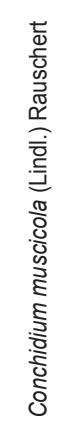 & 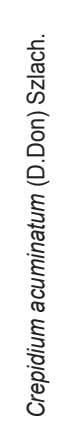 & 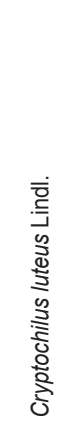 & 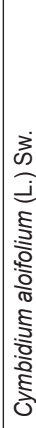 & 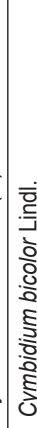 & 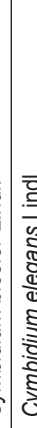 & 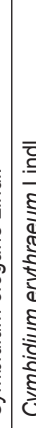 & 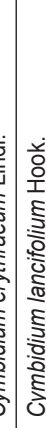 & 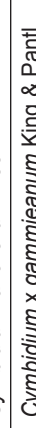 & 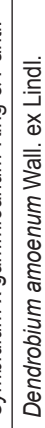 & 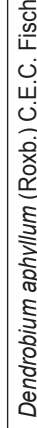 & 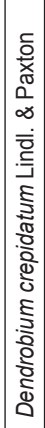 & 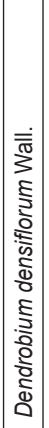 & 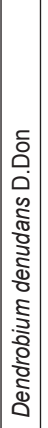 & 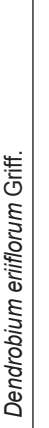 & 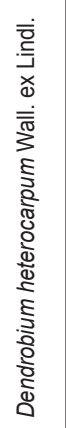 & 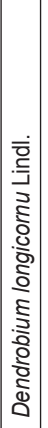 & 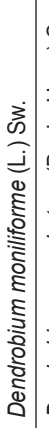 & 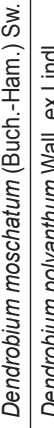 & 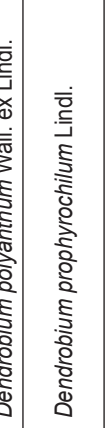 & 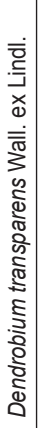 & 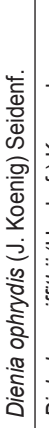 & 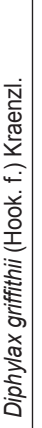 & 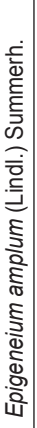 \\
\hline 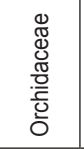 & 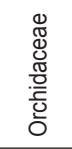 & 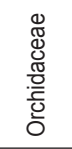 & 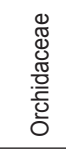 & 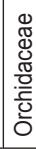 & 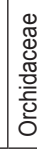 & $\begin{array}{l}\frac{\pi}{2} \\
0 \\
0 \\
\frac{\pi}{0} \\
0 \\
0 \\
0\end{array}$ & $\begin{array}{l}0 \\
\pi \\
d \\
\frac{\pi}{2} \\
\frac{c}{c} \\
c\end{array}$ & 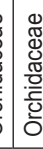 & $\begin{array}{l}\frac{a}{2} \\
0 \\
0 \\
\frac{\pi}{0} \\
\frac{0}{0} \\
0\end{array}$ & 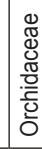 & 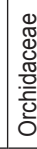 & 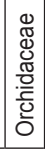 & 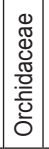 & 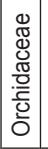 & 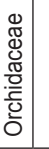 & 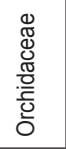 & \begin{tabular}{|l|} 
\\
$\mathbb{8}$ \\
$\mathbb{0}$ \\
$\frac{\pi}{0}$ \\
$\frac{0}{0}$ \\
0 \\
\end{tabular} & 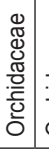 & 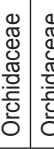 & 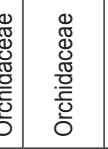 & 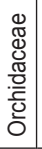 & 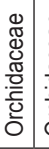 & 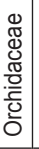 & 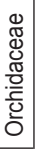 \\
\hline$\stackrel{\infty}{\text { cे }}$ & 守 & 哭 & 品 & స్ల్ల & હ्ల్ల & 岕 & เू & 岕 & 卢 & 离 & मिं & : & $\dot{\phi}$ & 心్లి & ह్ల & 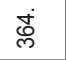 & 岕 & 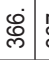 & $\begin{array}{l}\vec{e} \\
\vec{m}\end{array}$ & ஜ্ं & 忿 & 灾 & స్ & న్ \\
\hline
\end{tabular}




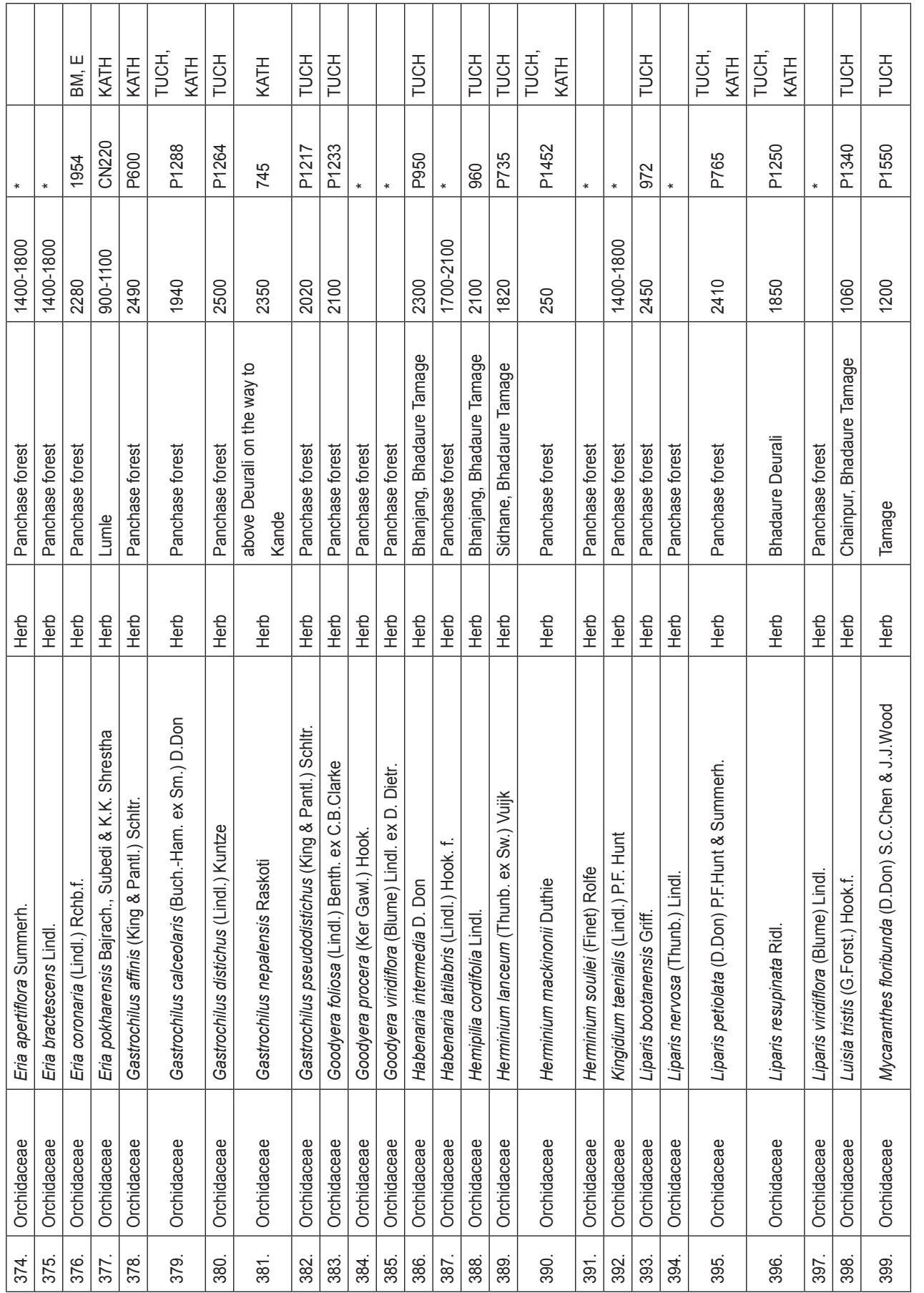


Bhandari et al.: A checklist of flowering plants of Panchase.

\begin{tabular}{|c|c|c|c|c|c|c|c|c|c|c|c|c|c|c|c|c|c|c|c|}
\hline & & 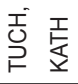 & 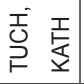 & 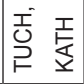 & 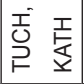 & 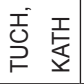 & 痩 & & 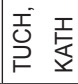 & $\begin{array}{l}\mathbf{I} \\
\mathbf{S} \\
\mathbf{S}\end{array}$ & 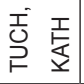 & $\begin{array}{l}\mathbf{I} \\
\mathbf{S} \\
\end{array}$ & $\begin{array}{l}\mathrm{I} \\
\mathrm{I} \\
\mathrm{P}\end{array}$ & 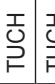 & $\begin{array}{r}\frac{1}{U} \\
\\
\end{array}$ & & 甹 & 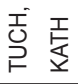 & \\
\hline * & * & $\begin{array}{l}\infty \\
\tilde{\alpha} \\
\Sigma\end{array}$ & $\stackrel{\bar{m}}{\grave{c}}$ & 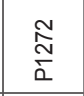 & 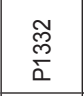 & 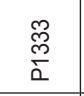 & 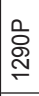 & * & $\begin{array}{l}\stackrel{N}{N} \\
\grave{\alpha}\end{array}$ & 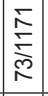 & $\underset{\mathbf{N}}{\bar{\Sigma}}$ & 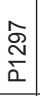 & $\begin{array}{l}\infty \\
\stackrel{N}{c} \\
\grave{c}\end{array}$ & 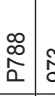 & $\begin{array}{c}\text { ç } \\
\text { c. }\end{array}$ & * & $\stackrel{\infty}{\stackrel{\infty}{\Sigma}}$ & $\stackrel{n}{\stackrel{n}{N}}$ & * \\
\hline & & 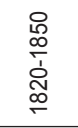 & $\begin{array}{l}\stackrel{\leftrightarrow}{+} \\
\stackrel{+}{\sim}\end{array}$ & $\stackrel{\circ}{\circ}$ & 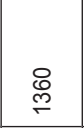 & $\stackrel{\text { 움 }}{\longrightarrow}$ & $\stackrel{8}{\grave{N}}$ & 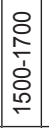 & $\begin{array}{l}\stackrel{\circ}{\circ} \\
\stackrel{\infty}{\leftarrow}\end{array}$ & 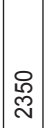 & 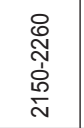 & 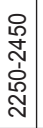 & $\frac{.}{N}$ & ঠి & $\begin{array}{c}\text { N. } \\
\text { N }\end{array}$ & & ণ্ণి & ஜ్రి & 음 \\
\hline 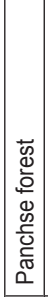 & 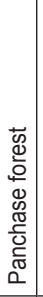 & 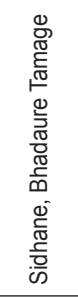 & 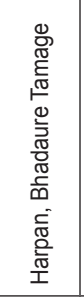 & 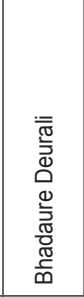 & 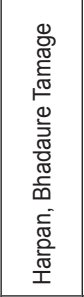 & 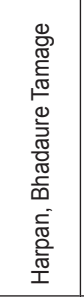 & 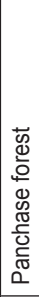 & 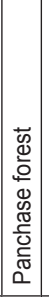 & 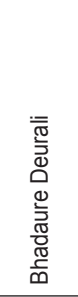 & 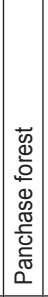 & 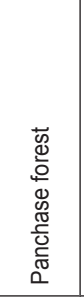 & 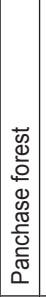 & 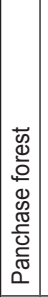 & 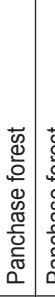 & 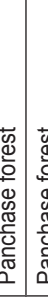 & 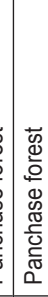 & 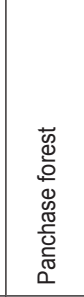 & 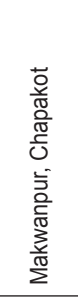 & 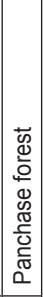 \\
\hline $\begin{array}{l}\frac{0}{0} \\
\frac{\overline{0}}{1}\end{array}$ & $\frac{\stackrel{0}{0}}{\frac{0}{1}}$ & $\begin{array}{l}\frac{0}{\overline{0}} \\
\frac{0}{1}\end{array}$ & $\begin{array}{l}\frac{0}{\overline{0}} \\
\frac{0}{1}\end{array}$ & $\begin{array}{l}\frac{0}{\frac{0}{\Phi}} \\
\frac{1}{1}\end{array}$ & $\begin{array}{l}\frac{0}{\overline{0}} \\
\frac{0}{1}\end{array}$ & $\begin{array}{l}\frac{0}{\overline{0}} \\
\frac{1}{1}\end{array}$ & $\frac{\frac{0}{0}}{\frac{0}{1}}$ & $\begin{array}{l}\frac{0}{\bar{\omega}} \\
\frac{1}{1}\end{array}$ & $\begin{array}{l}\frac{0}{\overline{0}} \\
\frac{1}{1}\end{array}$ & $\begin{array}{l}\frac{0}{\bar{\omega}} \\
\frac{\mathbf{\omega}}{1}\end{array}$ & $\frac{\frac{0}{0}}{\frac{0}{1}}$ & $\begin{array}{l}\frac{0}{\overline{0}} \\
\frac{1}{I}\end{array}$ & 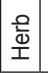 & $\frac{0}{\overline{0}} \frac{3}{1}$ & $\frac{0}{\frac{2}{0}}$ & $\frac{0}{\bar{\phi}}$ & $\frac{\frac{O}{0}}{\frac{0}{1}}$ & $\begin{array}{l}\frac{0}{0} \\
\frac{0}{1}\end{array}$ & $\frac{\circ}{\overline{0}}$ \\
\hline 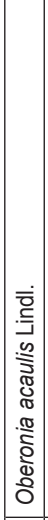 & 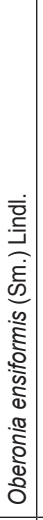 & 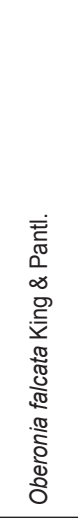 & 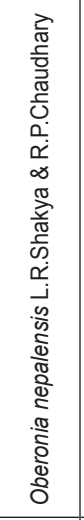 & 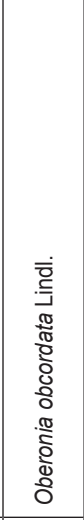 & 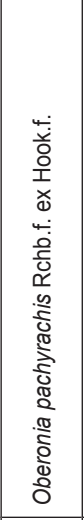 & 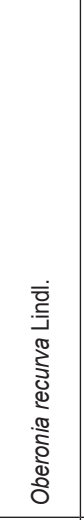 & 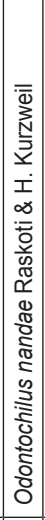 & 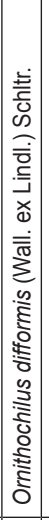 & 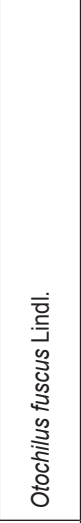 & 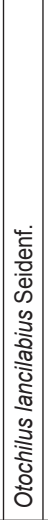 & 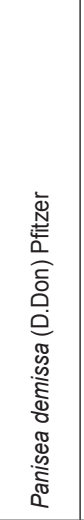 & 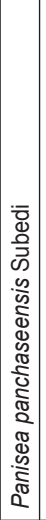 & 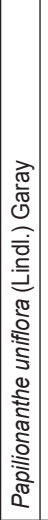 & 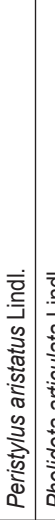 & 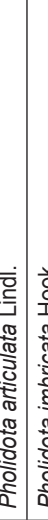 & 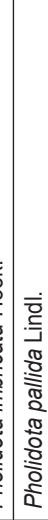 & 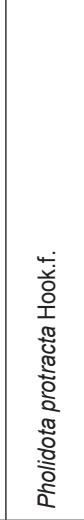 & 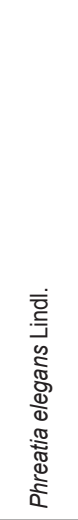 & 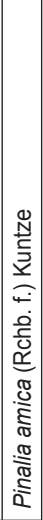 \\
\hline 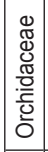 & 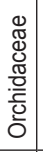 & 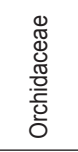 & 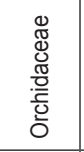 & 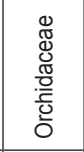 & 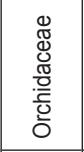 & 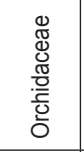 & 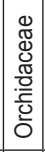 & 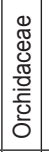 & 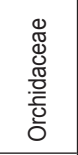 & 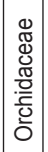 & 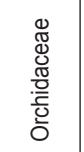 & 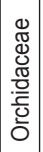 & 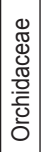 & 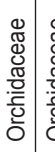 & 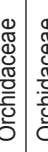 & 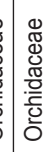 & 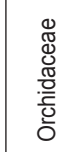 & 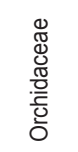 & 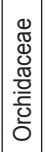 \\
\hline ৪্ণ & $\dot{\sigma}$ & ণิ & ơ & 字 & ஜீ & $\dot{\leftrightarrow}$ & 守 & $\begin{array}{l}\infty \\
q \\
\sigma\end{array}$ & ஓे & $\frac{\dot{v}}{\dot{\gamma}}$ & $\dot{\xi}$ & 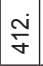 & $\stackrel{m}{q}$ & $\underset{\dot{r}}{\dot{\sigma}}$ & 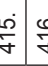 & $\stackrel{\tilde{\sigma}}{\dot{\gamma}}$ & $\stackrel{\infty}{\frac{\infty}{\gamma}}$ & $\stackrel{\circ}{\frac{\rho}{\gamma}}$ & ণ্் \\
\hline
\end{tabular}




\begin{tabular}{|c|c|c|c|c|c|c|c|c|c|c|c|c|c|c|c|c|c|c|c|c|c|c|}
\hline 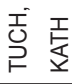 & $\begin{array}{l}\text { I } \\
\text { ㄱ }\end{array}$ & 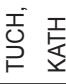 & $\begin{array}{l}\text { I } \\
\text { 간 }\end{array}$ & $\begin{array}{l}\text { İ } \\
\stackrel{\mathrm{U}}{\mathrm{E}}\end{array}$ & $\begin{array}{l}\mathrm{I} \\
\mathrm{S} \\
\mathrm{P}\end{array}$ & & $\begin{array}{l}\text { I } \\
\text { U } \\
\longmapsto\end{array}$ & $\begin{array}{l}\text { İ } \\
\stackrel{I}{上}\end{array}$ & $\begin{array}{l}\mathrm{I} \\
\mathrm{U} \\
\mathrm{P} \\
\end{array}$ & \begin{tabular}{|l|}
$\mathrm{I}$ \\
$\mathrm{U}$ \\
$\mathrm{P}$ \\
\end{tabular} & 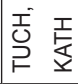 & 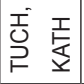 & $\begin{array}{l}I \\
\text { 焉 }\end{array}$ & 昰 & $\begin{array}{l}\text { I } \\
\mathrm{O} \\
\mathrm{P}\end{array}$ & $\begin{array}{l}\mathrm{I} \\
\mathrm{U} \\
\mathrm{P}\end{array}$ & $\begin{array}{l}\mathrm{I} \\
\mathrm{J} \\
\mathrm{P}\end{array}$ & \begin{tabular}{l}
$\mathrm{T}$ \\
$\mathrm{U}$ \\
\hdashline \\
\end{tabular} & $\begin{array}{l}\mathrm{T} \\
\mathrm{c} \\
\mathrm{F} \\
\mathrm{F}\end{array}$ & $\stackrel{\mathrm{I}}{\mathrm{O}}$ & $\begin{array}{l}\text { I } \\
\stackrel{2}{~}\end{array}$ & 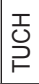 \\
\hline 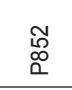 & ळో & 占 & 离 & 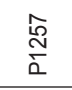 & ஃ & * & $\begin{array}{l}\stackrel{+}{\tilde{m}} \\
\stackrel{m}{\alpha} \\
\end{array}$ & $\begin{array}{l}\widetilde{N} \\
\stackrel{2}{\alpha}\end{array}$ & $\begin{array}{l}\mathscr{D} \\
\stackrel{D}{0} \\
\end{array}$ & 产 & 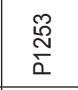 & $\stackrel{?}{\stackrel{?}{\Lambda}}$ & $\begin{array}{l}\mathscr{D} \\
\stackrel{0}{1} \\
\Sigma \\
\end{array}$ & $\bar{g}$ & $\begin{array}{l}1 \\
0 \\
0 \\
0 \\
\end{array}$ & $\sum_{\frac{\pi}{5}}^{\frac{\pi}{5}}$ & ڤે & & ఏ & 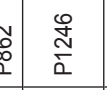 & 임 & 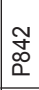 \\
\hline 吕 & ণ্ల్ & 怘 & ஜ్రి & 怘 & ষ্ల్ & ठ். & 荘 & 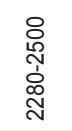 & 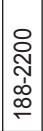 & 음 & 怘 & 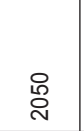 & 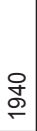 & ঃ & \begin{tabular}{|c|}
0 \\
0 \\
\hdashline
\end{tabular} \mid & 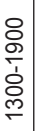 & $\begin{array}{l}\stackrel{\circ}{\circ} \\
-\end{array}$ & প্ণে & : & $\stackrel{\text { 怘 }}{\stackrel{一}{r}}$ & 导 & 응 \\
\hline 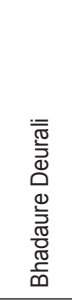 & 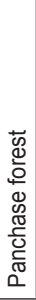 & 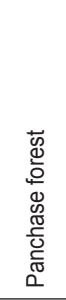 & 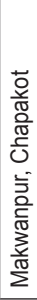 & $\begin{array}{l}\frac{\pi}{0} \\
\frac{\pi}{0} \\
\frac{\pi}{0} \\
\Phi \\
\mathbb{8} \\
\frac{\pi}{0} \\
\frac{0}{\pi} \\
0 \\
0\end{array}$ & 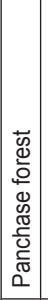 & 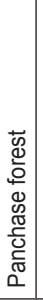 & 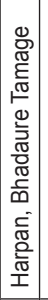 & 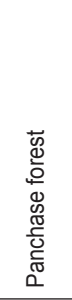 & 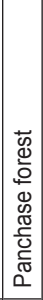 & 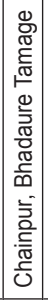 & 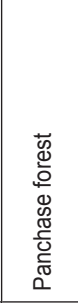 & 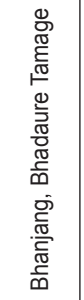 & 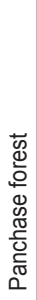 & 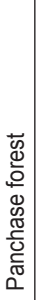 & 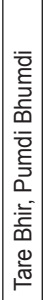 & 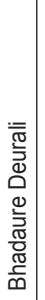 & 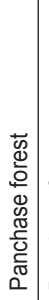 & 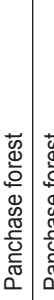 & 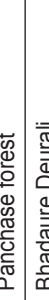 & 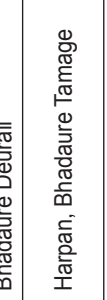 & 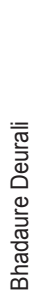 & 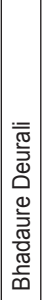 \\
\hline $\begin{array}{l}\frac{0}{\overline{0}} \\
\frac{1}{1}\end{array}$ & $\frac{\text { 음 }}{\frac{0}{1}}$ & $\begin{array}{l}\frac{0}{\overline{0}} \\
\frac{0}{1}\end{array}$ & $\begin{array}{l}\frac{0}{0} \\
\frac{0}{1}\end{array}$ & $\begin{array}{l}\frac{0}{\frac{0}{1}} \\
\frac{1}{1}\end{array}$ & $\begin{array}{l}\frac{0}{\overline{0}} \\
\frac{1}{I}\end{array}$ & $\begin{array}{l}\frac{0}{\overline{0}} \\
\frac{1}{I}\end{array}$ & \begin{tabular}{|l}
$\frac{0}{\overline{0}}$ \\
$\frac{\mathbf{\omega}}{1}$
\end{tabular} & $\begin{array}{l}\frac{0}{\overline{0}} \\
\frac{0}{1}\end{array}$ & $\frac{}{\frac{0}{10}}$ & \begin{tabular}{|l|}
$\frac{O}{0}$ \\
$\frac{\mathbf{w}}{1}$
\end{tabular} & $\begin{array}{l}\frac{0}{\overline{0}} \\
\frac{0}{1}\end{array}$ & $\begin{array}{l}\frac{0}{0} \\
\frac{0}{1}\end{array}$ & $\frac{}{\frac{0}{\Phi}}$ & $\frac{0}{\frac{0}{0}}$ & $\mid$\begin{tabular}{|l}
$\frac{0}{0}$ \\
$\frac{\bar{\omega}}{x}$
\end{tabular} & $\begin{array}{l}\frac{0}{0} \\
\frac{0}{1}\end{array}$ & 产 & $\begin{array}{lll}\frac{0}{0} \\
\end{array}$ & 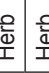 & $\begin{array}{l}\frac{0}{\frac{0}{1}} \\
\frac{1}{1}\end{array}$ & $\begin{array}{l}\frac{0}{\frac{0}{1}} \\
\frac{1}{1}\end{array}$ & $\frac{\text { 은 }}{\frac{0}{1}}$ \\
\hline 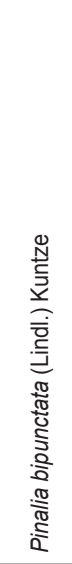 & 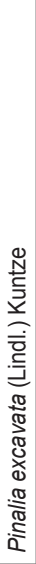 & 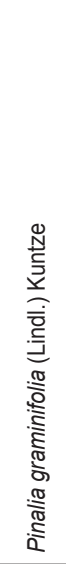 & 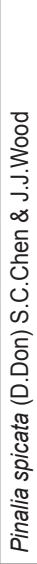 & 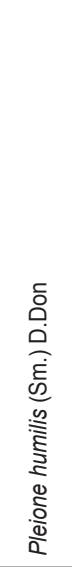 & 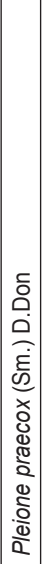 & 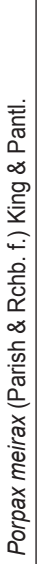 & 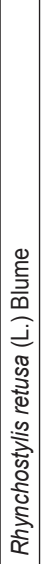 & 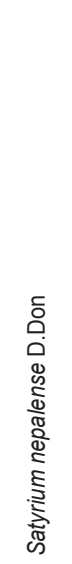 & 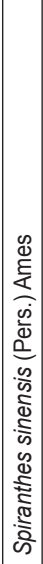 & 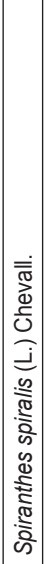 & 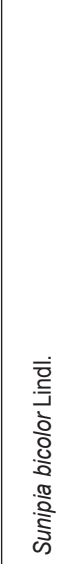 & 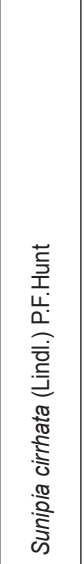 & 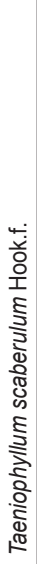 & 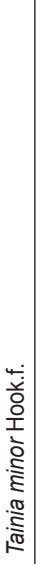 & 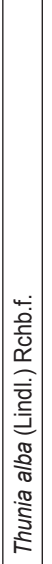 & 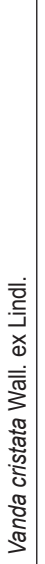 & 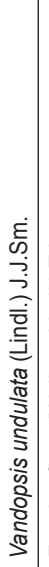 & 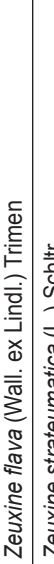 & 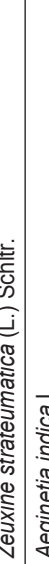 & 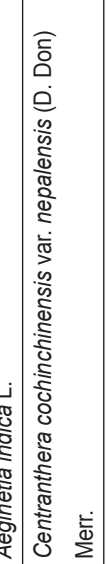 & 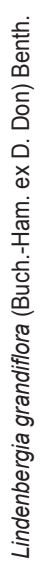 & 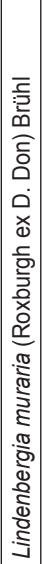 \\
\hline 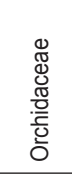 & 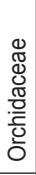 & 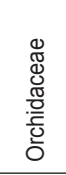 & 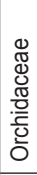 & 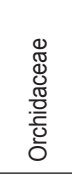 & 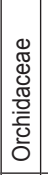 & 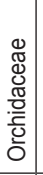 & 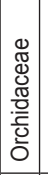 & 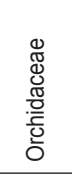 & 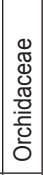 & 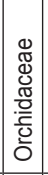 & 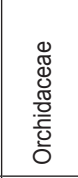 & 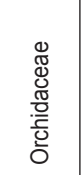 & 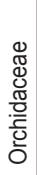 & 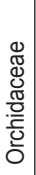 & 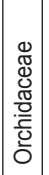 & $\begin{array}{l}\mathbb{\Xi} \\
\mathbb{\Phi} \\
\mathbb{\Xi} \\
\frac{\mathbb{Z}}{\bar{c}} \\
\overline{0} \\
0\end{array}$ & 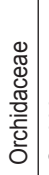 & 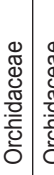 & 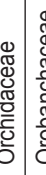 & 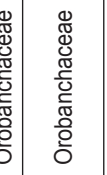 & 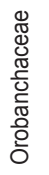 & 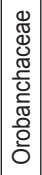 \\
\hline$\dot{\bar{y}}$ & สิ & $\stackrel{\overbrace \vartheta}{\Im}$ & $\underset{\Im}{*}$ & ભூ & 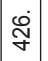 & $\stackrel{\Im}{\Im}$ & 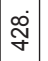 & $\stackrel{\mathbb{Y}}{\forall}$ & ஜ্ं & $\dot{\mathscr{m}}$ & ্ָণ & ঙ্ণ & 岀 & 子 & $\begin{array}{l}\dot{\theta} \\
\dot{\vartheta}\end{array}$ & 宇 & 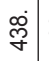 & প্ষেণ & \& & ปู & ஓं & 过 \\
\hline
\end{tabular}




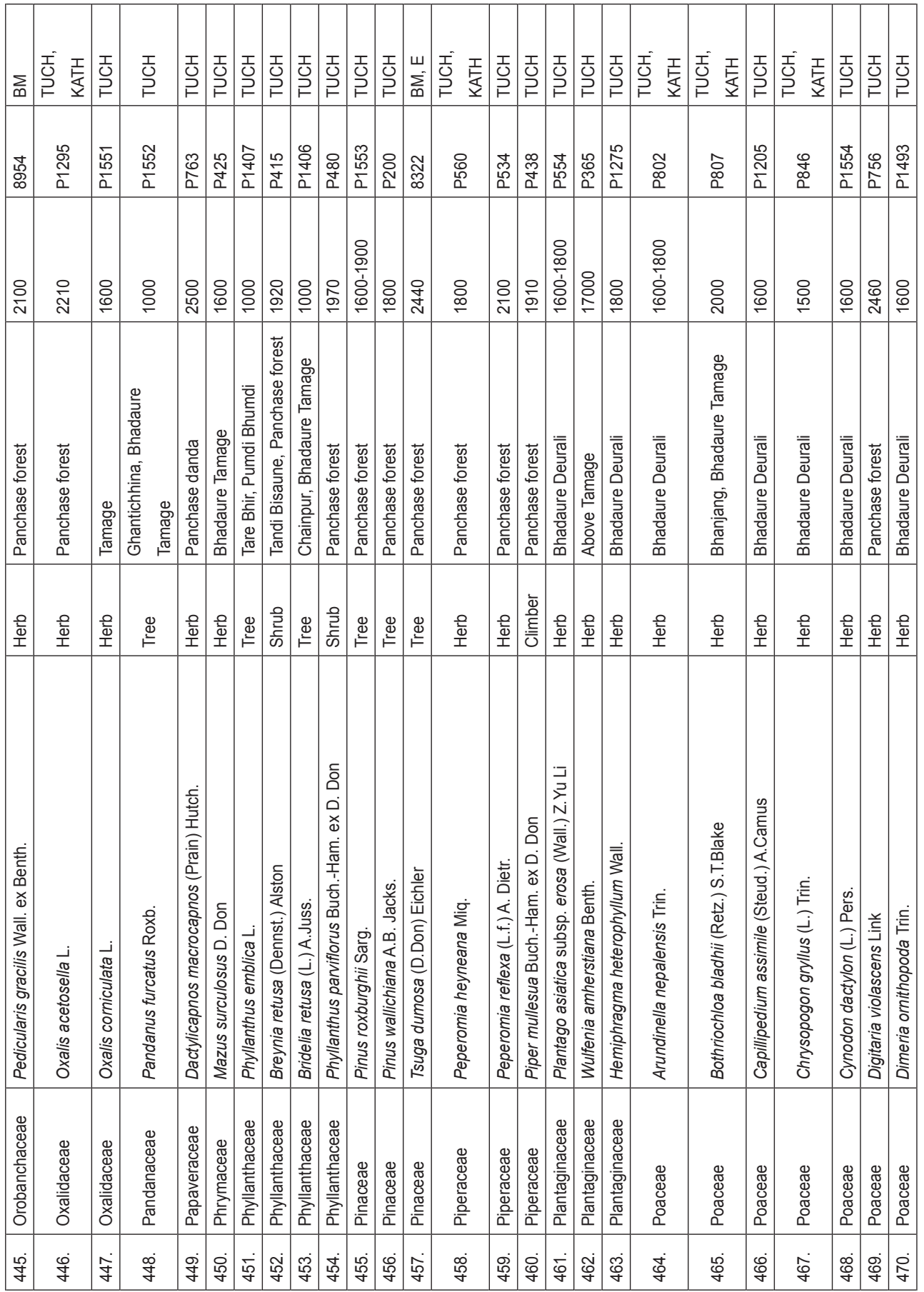




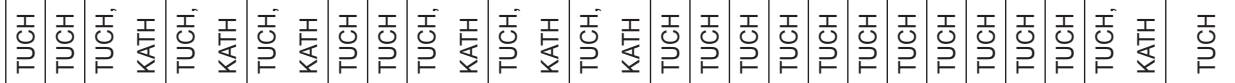

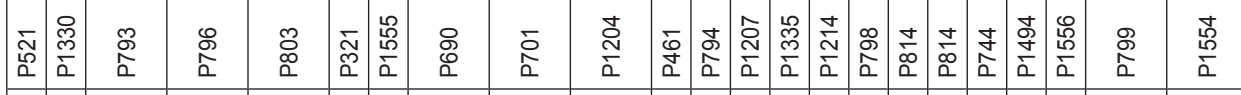

유ํ

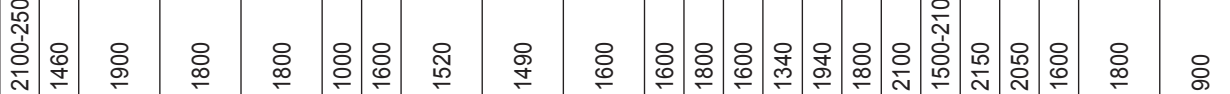

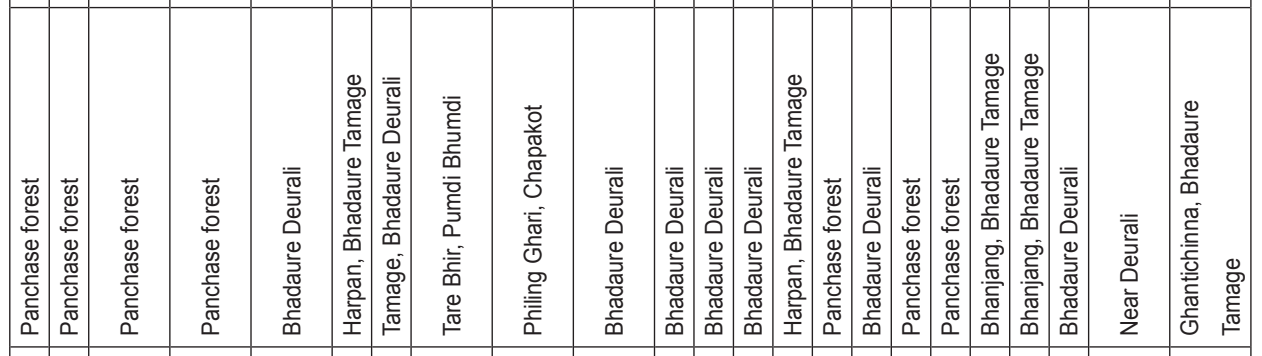

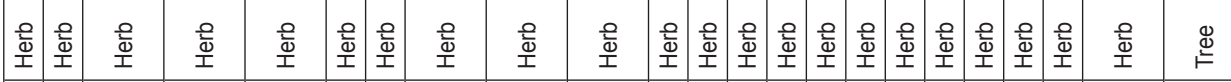

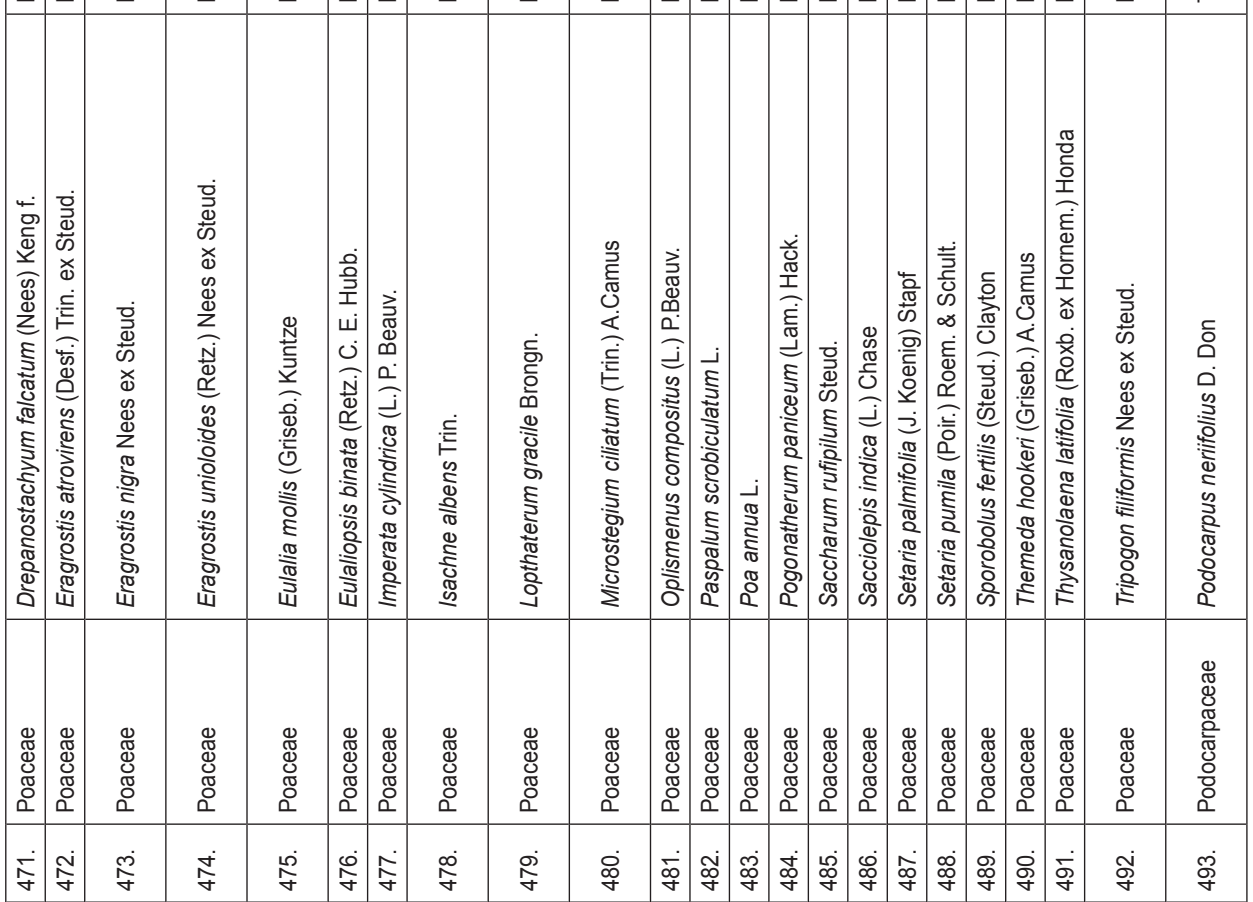




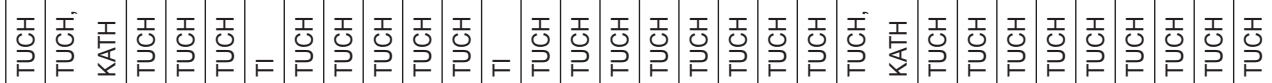

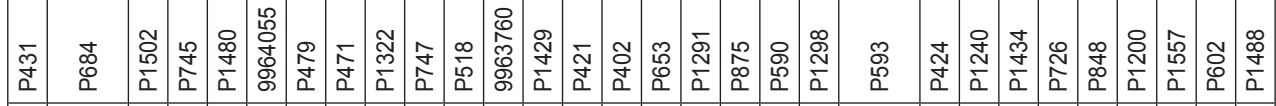
空

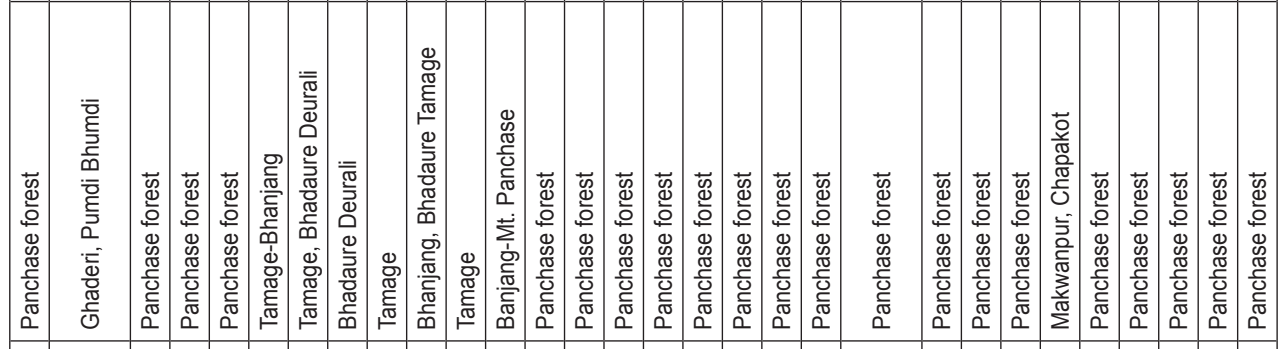

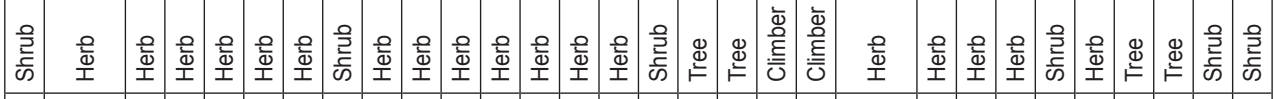

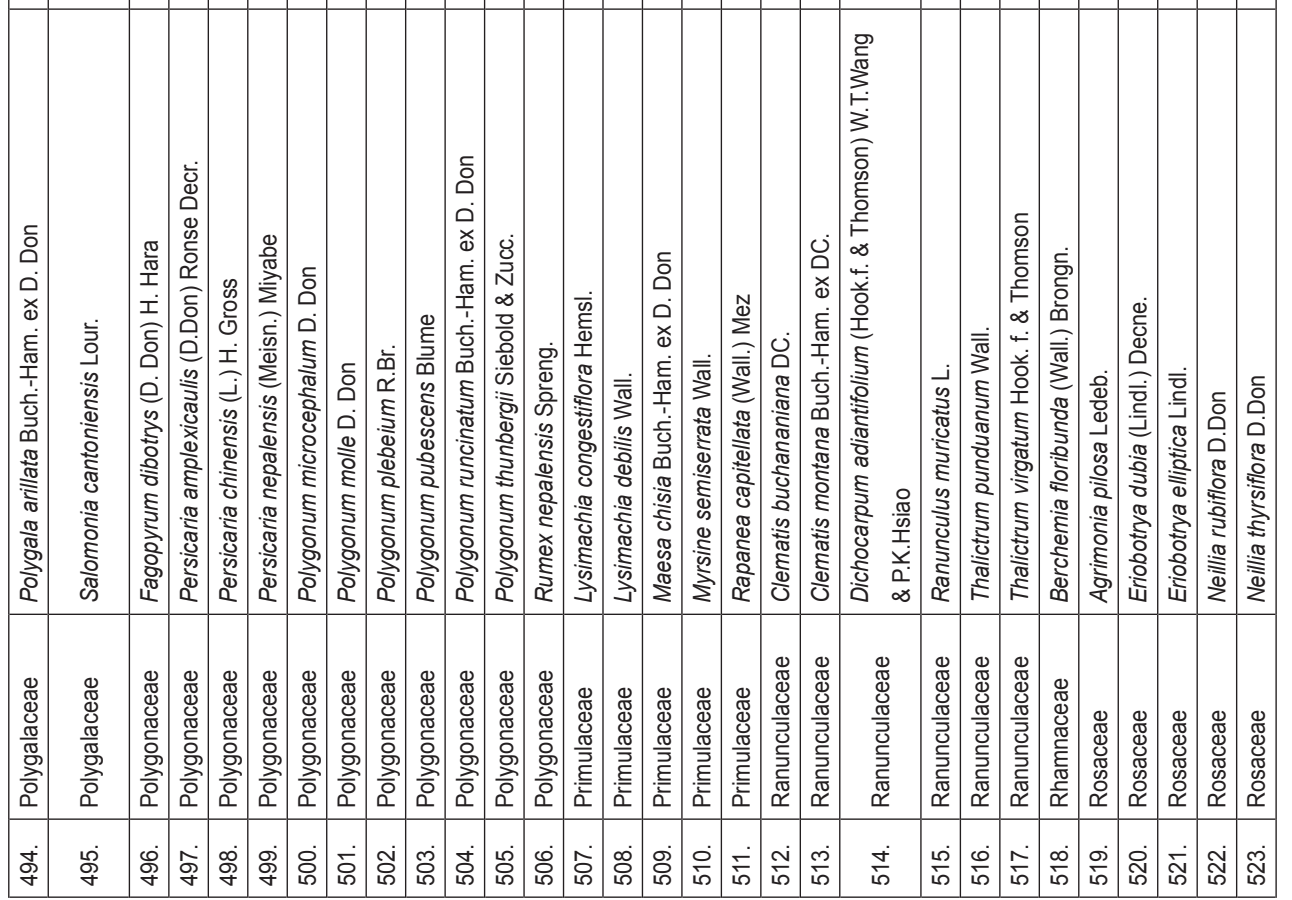




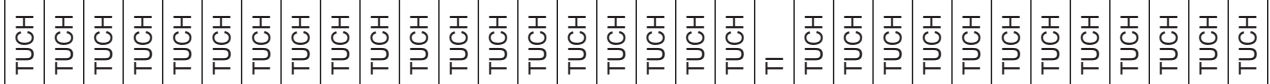

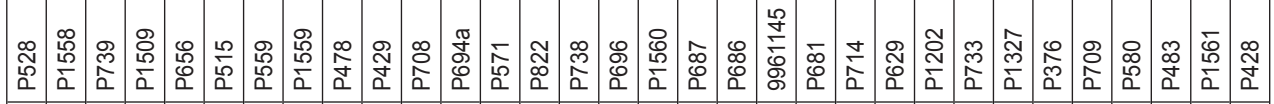

สั

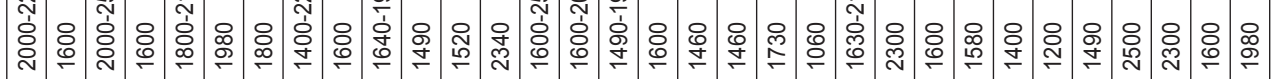

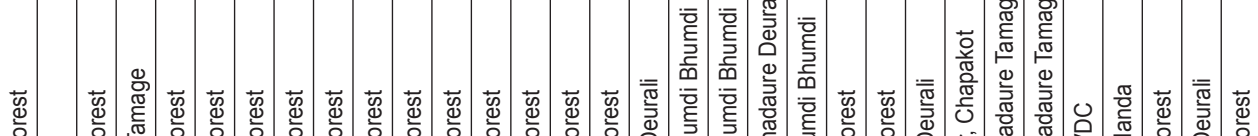

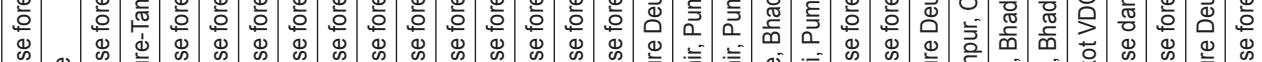

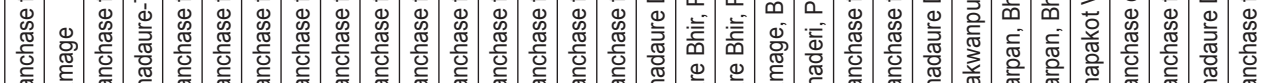

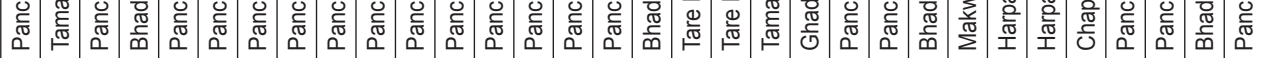

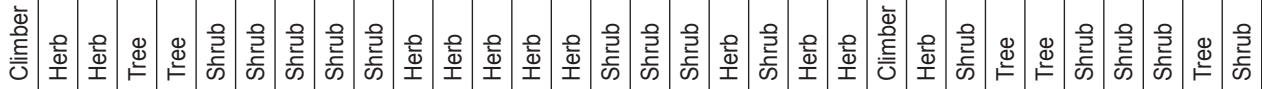

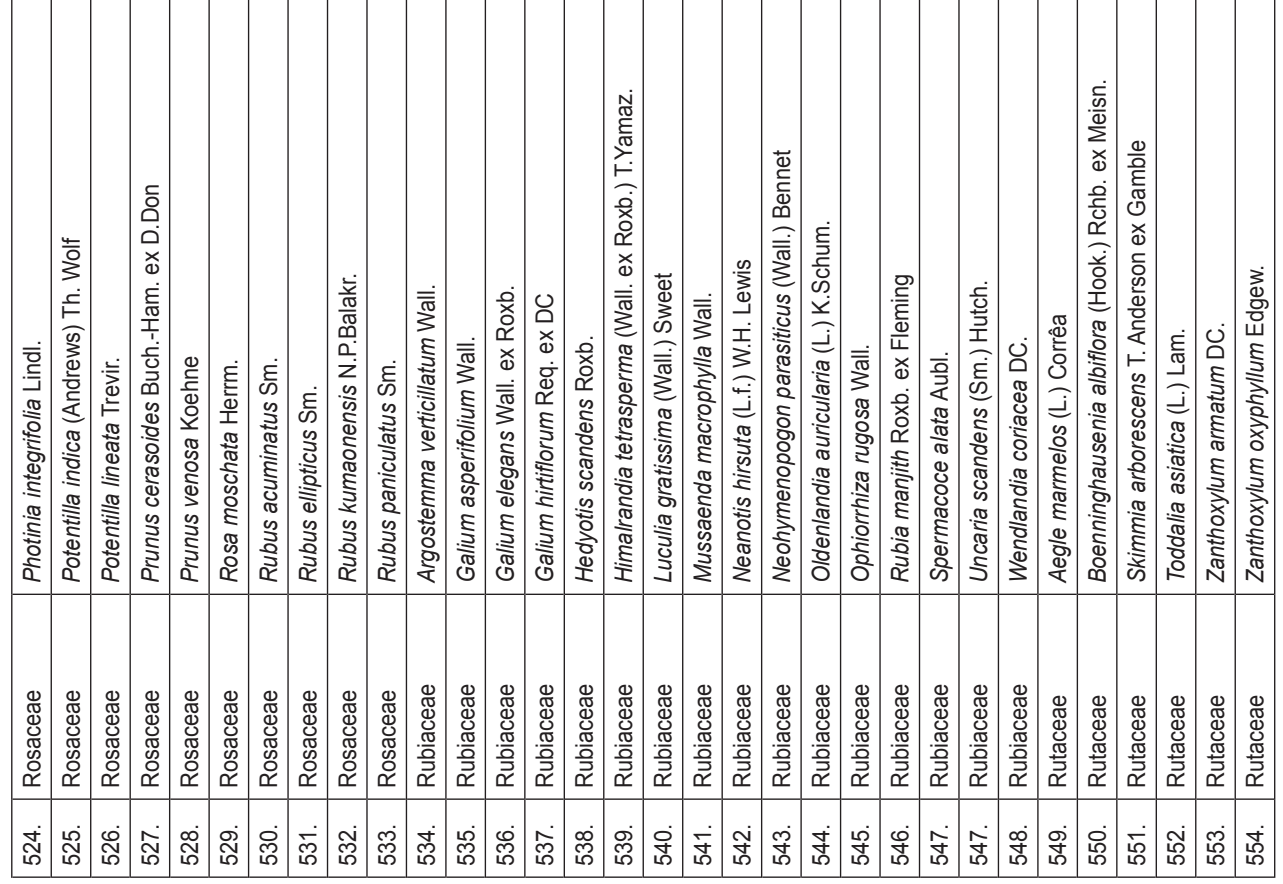




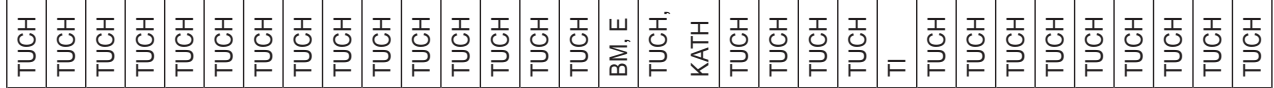

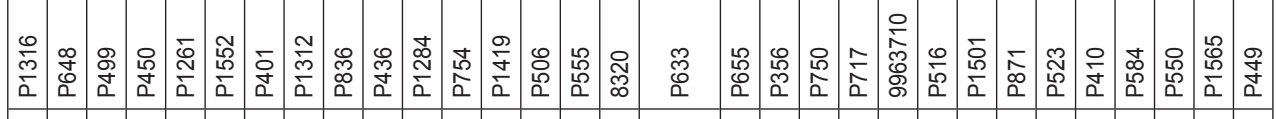

ะี้

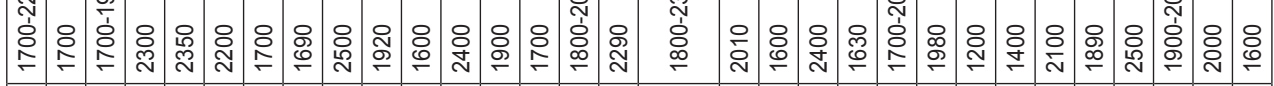

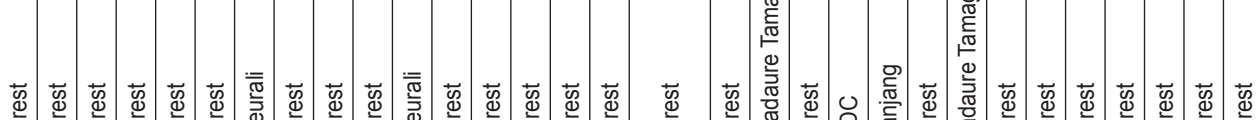

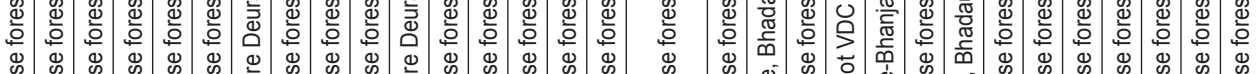

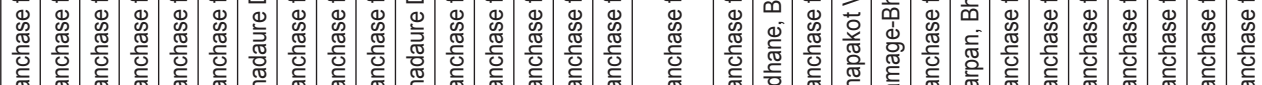

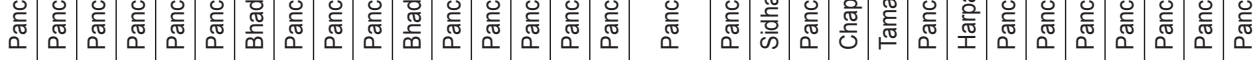

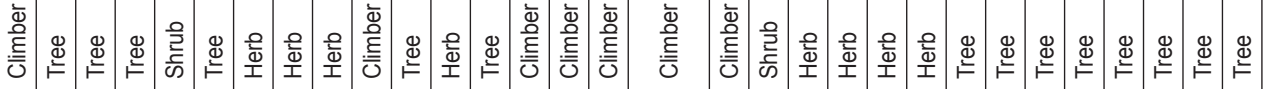

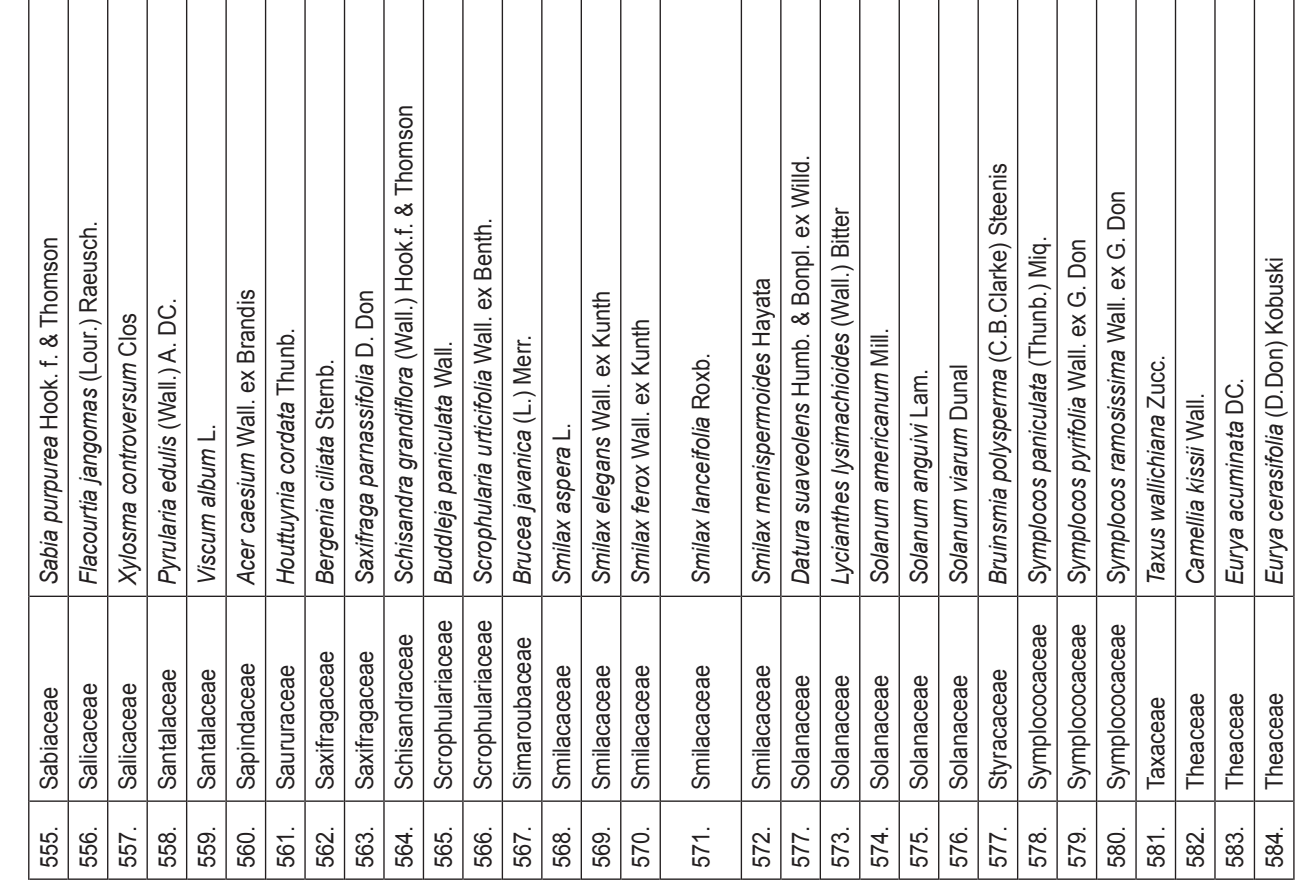




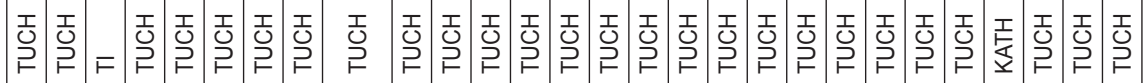

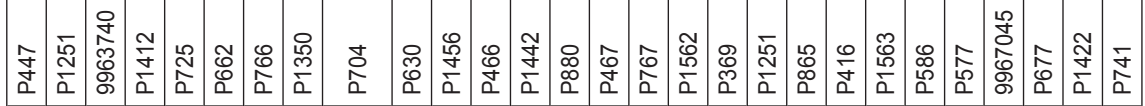

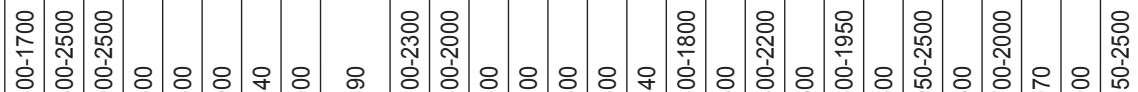

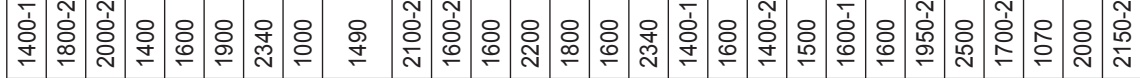

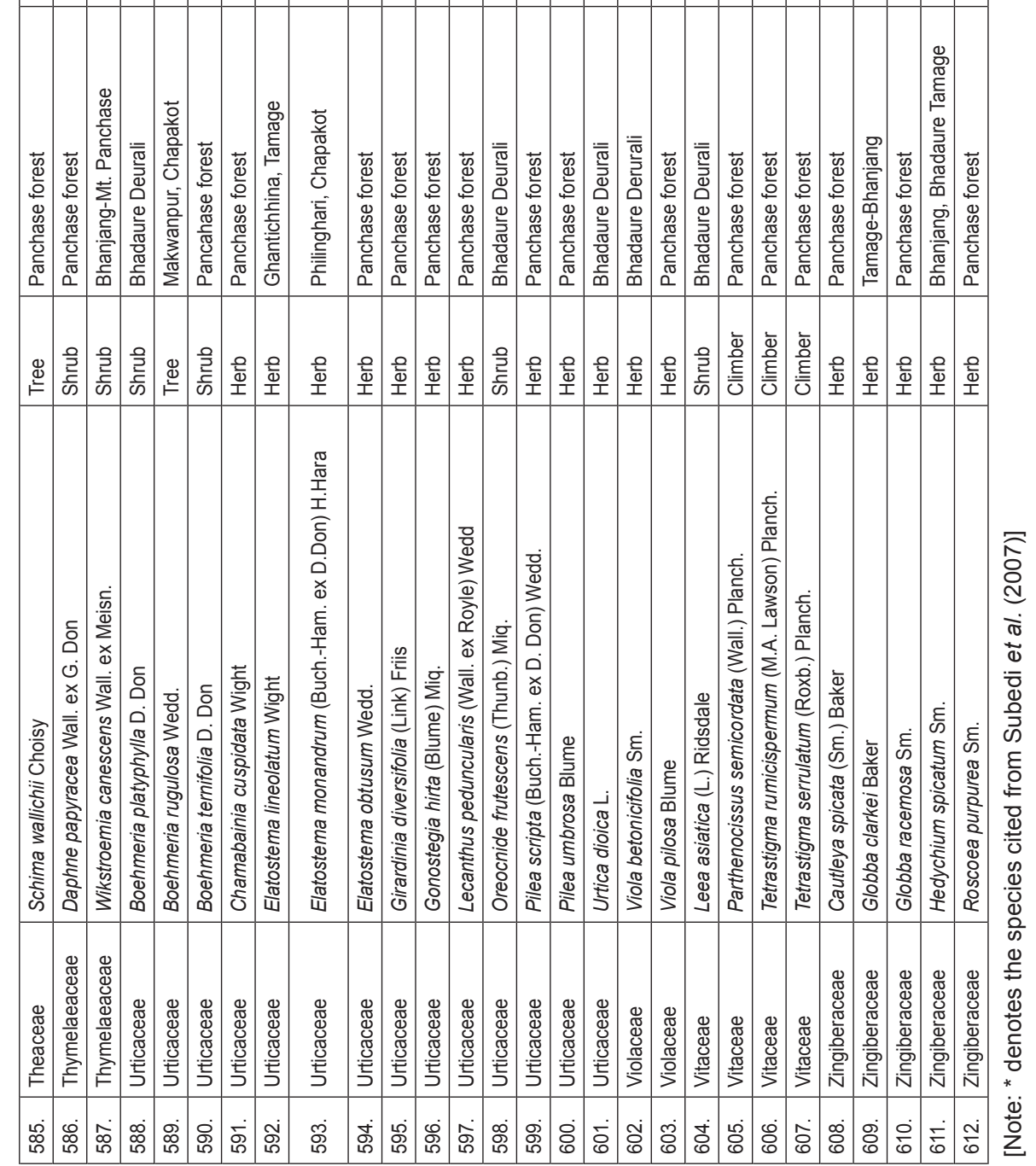

